\title{
Auswirkungen verkürzter Ruhezeiten auf Gesundheit und Work-Life-Balance bei Vollzeitbeschäftigten: Ergebnisse der BAuA-Arbeitszeitbefragung 2017
}

\author{
Nils Backhaus ${ }^{1} \cdot$ Corinna Brauner $^{1} \cdot$ Anita Tisch $^{1}$ \\ Online publiziert: 28. Oktober 2019 \\ ○ Der/die Autor(en) 2019, korrigierte Publikation 2020
}

\section{Zusammenfassung}

Flexible Arbeitszeitmodelle gewinnen in der modernen Arbeitswelt zunehmend an Bedeutung. Vielfach wird in Frage gestellt, ob bestehende Regelungen des Arbeitszeitgesetzes den Wandel der Arbeitswelt noch angemessen berücksichtigen. Vor diesem Hintergrund wurde auch eine Diskussion über die gesetzliche Mindestruhezeit von elf Stunden zwischen zwei Arbeitstagen angestoßen. Dieser Beitrag zeigt auf Basis der BAuA-Arbeitszeitbefragung 2017 (n=6136 Vollzeitbeschäftigte), dass die Verkürzung von Ruhezeiten, über alle Berufe hinweg, negativ mit psychosomatischen Beschwerden und Work-Life-Balance assoziiert ist. Zudem kann gezeigt werden, dass der vielfach bestätigte negative Zusammenhang zwischen täglichen Arbeitszeiten bzw. Überstunden mit Gesundheit und Work-Life-Balance zum Teil auf verkürzte Ruhezeiten zurückzuführen ist. Aus den Analysen wird deutlich, dass bereits eine gelegentliche Unterschreitung der elfstündigen Mindestruhezeit mit Risiken für Gesundheit und einer schlechteren Work-Life-Balance einhergeht.

Praktische Relevanz: Verkürzte Ruhezeiten zeigen negative Zusammenhänge zu Gesundheit und Work-Life-Balance. Die gesetzlichen Ruhezeiten stellen aus Sicht des Arbeitsschutzes daher einen notwendigen Mindeststandard dar, dessen Einhaltung für eine gesunde Arbeitszeitgestaltung für alle Tätigkeiten maßgeblich ist. Eine Flexibilisierung der Arbeitszeit, in Form einer Absenkung der Mindestruhezeit, ist aus arbeitswissenschaftlicher Sicht abzulehnen. Darüber hinaus sollten die bestehenden Ausnahmeregelungen kritisch reflektiert werden. Bei der Gestaltung und Bewertung von Arbeitszeiten sollte neben der Länge der Arbeitszeit stets auch die Ruhezeit berücksichtigt werden. Eine Unterschreitung der Mindestruhezeit sollte in jedem Fall vermieden werden.

Schlüsselwörter Arbeitszeit · BAuA-Arbeitszeitbefragung · Gesundheit · Verkürzte Ruhezeiten · Work-Life-Balance

Dr. Nils Backhaus

backhaus.nils@baua.bund.de

1 Gruppe „Wandel der Arbeit“, Bundesanstalt

für Arbeitsschutz und Arbeitsmedizin,

Friedrich-Henkel-Weg 1-25, 44139 Dortmund, Deutschland 


\title{
Quick returns, health, and work-life-balance in full-time employees: Results from the BAuA Working Time Survey 2017
}

\begin{abstract}
Flexible working time arrangements are becoming increasingly important in the modern world of work. It is often questioned whether existing working time regulations adequately consider the changes in the working world. Against this background, statutory minimum rest periods of eleven hours between two working days are challenged. Based on the BAuA Working Time Survey (2017) of $n=6136$ full-time employees, this article shows that the shortening of rest periods (quick returns) is negatively associated with psychosomatic health complaints and work-life balance across all occupations. The negative correlation between daily working hours or overtime with health and work-life balance is also partly attributable to quick returns. The analyses show that even occasional violations of the 11-hour minimum daily rest period are associated with health risks and a poor work-life balance.

Practical Relevance: Quick returns show negative correlations with health and work-life balance. Hence, minimum daily rest periods represent a necessary minimum standard for occupational safety and health. Flexibilisation of working hours, which shortens or interrupts the minimum daily rest periods, should be rejected to ensure organizational safety and health. Moreover, the existing derogations might also be revisited. When designing and evaluating working times, the length of rest periods should be taken into account in addition to working time duration. Quick returns should be avoided as far as possible.
\end{abstract}

Keywords BAuA Working Time Survey $\cdot$ Health $\cdot$ Quick returns $\cdot$ Short rest periods $\cdot$ Working time $\cdot$ Work-life balance

\section{Einleitung}

Ruhezeiten stellen den Zeitraum zwischen dem Ende der täglichen Arbeitszeit und der Wiederaufnahme der Tätigkeit am Folgetag dar (Zijlstra und Sonnentag 2006) und dienen zur langfristigen Regeneration sowie der Rückstellung der physischen und psychischen Beanspruchungen (Schlick et al. 2018). Gesetzlich vorgeschriebene Ruhezeiten dienen der Regeneration von Beschäftigten und bieten Zeit für soziale und kulturelle Aktivitäten. Arbeitswissenschaftlich betrachtet sind Ruhezeiten, ähnlich wie Pausen als regelmäßige Arbeitsunterbrechungen während der Arbeitszeit (Wendsche und Lohmann-Haislah 2016), von großer Bedeutung. Während der Unterbrechung werden durch die Tätigkeit in Anspruch genommene Ressourcen wiederhergestellt und die arbeits- bzw. tageszeitbedingte Ermüdung wird ausgeglichen (Sonnentag und Natter 2004). Ist nach Belastungen eine ausreichende Erholung über einen längeren Zeitraum nicht möglich, kann die Folge eine Verlustspirale sein, im Zuge derer physische und psychische Ressourcen nach und nach abgebaut werden (Sonnentag und Fritz 2007). Folglich ist Erholung ein wichtiger Prozess, der Energiereserven wieder auffüllt und so langfristig zum Erhalt der Gesundheit und Arbeitsfähigkeit beiträgt (Meijman und Mulder 1998). Zum Schutz der Sicherheit und Gesundheit von Arbeitnehmerinnen und Arbeitnehmern sieht die Arbeitszeitgesetzgebung ${ }^{1}$ deshalb eine tägliche Ruhezeit

${ }^{1}$ EU-Arbeitszeitrichtlinie (2003/88/EG, Art. 3) bzw. Arbeitszeitgesetz (ArbZG § 5). von mindestens elf aufeinanderfolgenden Stunden zwischen zwei Arbeitseinsätzen vor.

In einer sich wandelnden Arbeitswelt wird vermehrt eine flexible Gestaltung der Arbeitszeit gefordert. Neben einer Ausdehnung der täglichen Höchstarbeitszeiten wird hierbei - insbesondere von Arbeitgeberverbänden - auch eine mögliche Aufteilung, Unterbrechung bzw. Verkürzung der Ruhezeiten zur Diskussion gestellt (BDA 2015; Börkircher und Walleter 2018). Begründet werden die Forderungen mit zunehmenden Flexibilitätsanforderungen, die sich durch einen erhöhten Wettbewerbsdruck ergeben. Nicht selten werden aber auch Beschäftigtenwünsche angeführt, z. B. um Arbeit und Privatleben besser vereinbaren zu können (BDA 2015). Inwiefern derartige Arbeitszeitmodelle tatsächlich zu einer besseren Vereinbarkeit von Arbeit und Privatleben bzw. Work-Life-Balance führen ist fraglich. Ebenso bleibt aus arbeitswissenschaftlicher Perspektive unklar, welche Auswirkungen sich für die Gesundheit von Beschäftigten ergeben. Dies liegt nicht zuletzt daran, dass Ruhezeiten in breit angelegten Beschäftigtenbefragungen in Deutschland bislang nicht berücksichtigt wurden. Aus diesem Grund wurden in der BAuA-Arbeitszeitbefragung 2017 Beschäftigte erstmalig zur Einhaltung ihrer Mindestruhezeiten befragt. Der vorliegende Beitrag stellt erste Analysen zum Zusammenhang zwischen der Verkürzung von Ruhezeiten und der Work-Life-Balance dar. Da anzunehmen ist, dass verkürzte oder unterbrochene Ruhezeiten einer ausreichenden Erholung entgegenwirken und sich langfristig auch nachteilig auf die Gesundheit von Beschäftigten auswirken, wird ebenso auch der Zusammenhang zwischen verkürzten bzw. geteilten Ruhezeiten und psychosomatischen Be- 
schwerden von Beschäftigten betrachtet. In den Analysen wird außerdem das Zusammenwirken von verkürzten $\mathrm{Ru}$ hezeiten mit der Länge der Arbeitszeiten sowie mit Überstunden berücksichtigt, um zwischen Ruhezeitverletzungen aufgrund einer Aufteilung bzw. Verschiebung der regulären täglichen Arbeitszeit oder aber aufgrund von (über-)langen Arbeitszeiten zu differenzieren.

Im folgenden Abschnitt werden bisherige Erkenntnisse zu den Auswirkungen verkürzter Ruhezeiten zusammengefasst. Die BAuA-Arbeitszeitbefragung sowie die Analysemethoden werden in Kapitel drei dargestellt. AnschlieBend werden die Ergebnisse der empirischen Analyse des Zusammenhangs zwischen verkürzten Ruhezeiten, Gesundheit und Work-Life-Balance, unter besonderer Berücksichtigung des Zusammenwirkens von verkürzten Ruhezeiten mit langen Arbeitszeiten und Überstunden dargestellt. Abschließend erfolgt eine Diskussion und kritische Reflexion der Ergebnisse.

\section{Stand der Forschung und Fragestellungen}

Bisherige Studien weisen bereits darauf hin, dass verkürzte Ruhezeiten kurzfristig zu einer Reduzierung der Schlaflänge und Schlafqualität führen (Vedaa et al. 2016, 2017a), was sich in einer erhöhten Müdigkeit bzw. Schläfrigkeit am Folgetag niederschlägt (Eldevik et al. 2013). Langfristig sind Schlafstörungen und Erschöpfungssyndrome (Åkerstedt und Kecklund 2017; Dahlgren et al. 2016; Härmä et al. 2018) sowie ein erhöhtes Risiko für eine Arbeitsunfähigkeit durch Krankheit (Vedaa et al. 2017b) zu beobachten. Negative Auswirkungen ergeben sich auch für das Unfallrisiko und die Fehlerrate (Folkard und Lombardi 2006; Nielsen et al. 2019; Vedaa et al. 2019). Dadurch kann es nicht nur zur Gefährdung der Beschäftigten selbst kommen, sondern auch zu Risiken für unbeteiligte Dritte, wie z.B. Patientinnen und Patienten (Bernstrøm et al. 2019; Vedaa et al. 2019). Neben gesundheitlichen Risiken betrachten weitere Studien auch mögliche Auswirkungen auf die Vereinbarkeit und Work-Life-Balance von Beschäftigten. So scheinen verkürzte Ruhezeiten die Konflikte zwischen Berufs- und Privatleben zu verursachen und mit Einschränkungen der Vereinbarkeit von Privatleben und Beruf einherzugehen (Åkerstedt und Kecklund 2017; Karhula et al. 2018, 2017).

Die überwiegende Mehrheit der Studien zu den Auswirkungen von verkürzten Ruhezeiten fokussiert auf Schichtarbeitende und dabei vorwiegend auf Beschäftigte im Krankenhaus. Die Frage nach der Übertragbarkeit der bisherigen Erkenntnisse auf eine breite Erwerbsbevölkerung bleibt bislang weitgehend unbeantwortet. In dem vorliegenden Beitrag soll deshalb, repräsentativ für alle abhängig Beschäftigten in Deutschland, der Frage nachgegangen werden, in welchem Zusammenhang verkürzte Ruhezeiten mit der Work-Life-Balance sowie mit psychosomatischen Beschwerden von Beschäftigten stehen.

Die Vermutung liegt nahe, dass verkürzte Ruhezeiten häufig mit langen oder sehr langen Arbeitszeiten einhergehen. Bei einer Ausweitung der Arbeitszeiten müsste der Arbeitsbeginn am nächsten Tag verschoben werden, um eine relative Verkürzung von Ruhezeiten zu vermeiden. Über die Auswirkungen langer Arbeitszeiten und Überstunden auf Sicherheit und Gesundheit bei der Arbeit liegen bereits vielfältige Erkenntnisse vor. Immer wieder zeigen repräsentative Studien, dass lange Arbeitszeiten mit schlechterer physischer und psychischer Gesundheit (z.B. Müller et al. 2018) und negativen Auswirkungen für die Work-Life-Balance einhergehen (BAuA 2016). Weitere Erkenntnisse stützen sich auf eine Vielzahl von Längsschnitt- und Kohortenstudien sowie umfangreiche Literaturreviews. Es wird deutlich, dass sich bei langen Arbeitszeiten beispielsweise das Risiko für kardiovaskuläre Erkrankungen und Schlaganfälle (z. B. Kivimäki et al. 2015, 2017) oder Typ-2-Diabetes (z.B. Gilbert-Ouimet et al. 2018) erhöht. Außerdem nehmen gesundheitsschädliche Verhaltensweisen wie übermäßiger Alkoholkonsum (z.B. Virtanen et al. 2015) oder falsche Ernährung mit Folgen wie Übergewicht und Fettleibigkeit (z.B. Kim et al. 2016) zu. Zudem sind Schlafprobleme und psychische Beschwerden wie Depressionen oder Burnoutsymptome mit langen Arbeitszeiten assoziiert (z.B. Virtanen et al. 2018). Auch bezüglich des Einflusses langer Arbeitszeiten auf das Privatleben liegt bereits eine Vielzahl an Erkenntnissen vor. So kann davon ausgegan-
Abb. 1 Forschungsmodell mit verkürzten Ruhezeiten als Mediator (durchgezogene Linien stellen direkte Effekte dar, gestrichelte Linien verbildlichen die Mediation über Ruhezeiten) Fig. 1 Research model including quick returns as mediator (solid lines direct effects, dashed lines mediation by quick returns)

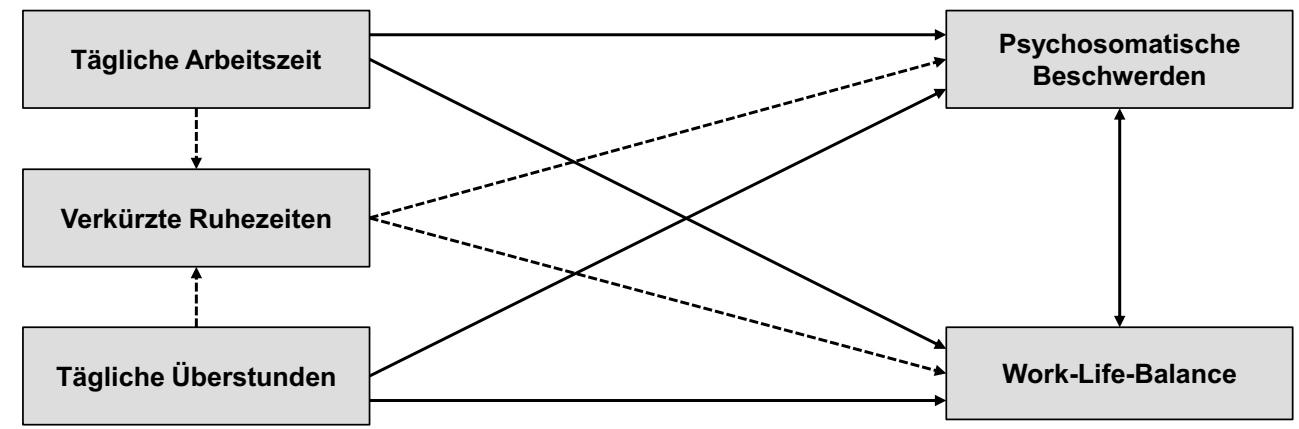


Abb. 2 Forschungsmodell mit Interaktion von verkürzten $\mathrm{Ru}$ hezeiten und den ArbeitszeitVariablen (durchgezogene Linien: direkte Effekte, gestrichelte Linien: Interaktion)

Fig. 2 Research model assuming an interaction of working time variables and quick returns (solid lines: direct effects, dashed lines: interaction)

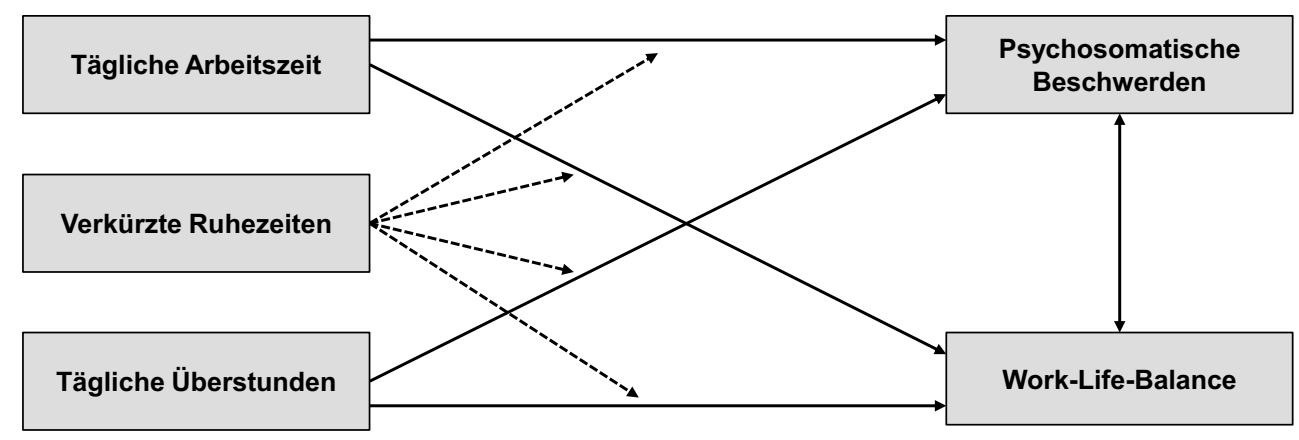

gen werden, dass lange Arbeitszeiten bzw. Überstunden die Zeit für familiäre und soziale Kontakte sowie nicht-berufsbezogene Tätigkeiten (Presser 2005; Wirtz 2010) und die Zufriedenheit mit der Work-Life-Balance reduzieren (Beermann et al. 2017; Fagan et al. 2012; Karhula et al. 2018, 2017; Ng und Feldman 2008).

In den folgenden Analysen zu den Auswirkungen von verkürzten Ruhezeiten wird zunächst davon ausgegangen, dass sich lange Arbeitszeiten bzw. viele Überstunden negativ auf den Gesundheitszustand und die Work-Life-Balance auswirken. Meist wird implizit angenommen, dass eine längere Arbeitszeit und eine hohe Anzahl von Überstunden das Risiko für verkürzte Ruhezeiten ansteigen lassen. Diese Annahme wurde bislang jedoch noch nicht empirisch überprüft. In der nachfolgenden Analyse wird daher zunächst ein Mediationsmodell untersucht, welches davon ausgeht, dass verkürzte Ruhezeiten auch durch die Länge der Arbeitszeit und Überstunden beeinflusst werden und den Effekt auf die Gesundheit bzw. Work-Life-Balance vermitteln. Die indirekten und direkten Effekte des Mediationsmodells sind grafisch in Abb. 1 veranschaulicht.

Insbesondere im Rahmen der Diskussion um flexible Arbeitszeitgestaltung wird eine Verkürzung der Ruhezeiten jedoch nicht zur Verlängerung der Arbeitszeiten gefordert, sondern um die Arbeit flexibel (z.B. am Nachmittag) unterbrechen zu können, um sie später (am Abend) wiederaufzunehmen. Insgesamt sollte hierdurch die Gesamtlänge der täglichen Arbeits- und Ruhezeit nicht beeinflusst werden. Um das Zusammenwirken von Arbeitszeitlänge bzw. Überstunden und verkürzten Ruhezeiten näher zu betrachten, wird im Folgenden deshalb zusätzlich ein Interaktionsmodell berücksichtigt. Stehen verkürzten Ruhezeiten nur in Kombination mit langen Arbeitszeiten bzw. Überstunden in einem negativen Zusammenhang mit Gesundheit bzw. Work-Life-Balance, so kann angenommen werden, dass nicht die Länge der Ruhezeit, sondern die Länge der Arbeitszeit bzw. die Überstunden die Work-Life-Balance und die Gesundheit beeinflussen. Das Interaktionsmodell ist in Abb. 2 dargestellt.

Die beschriebenen Annahmen werden anhand der Daten von Vollzeitbeschäftigten aus der BAuA-Arbeitszeitbefra- gung 2017 (Häring et al. 2018) untersucht. Im Folgenden werden die Stichprobe, die Operationalisierung der Variablen sowie die Vorgehensweise bei der Datenanalyse vorgestellt.

\section{Methode}

\subsection{Stichprobe}

Für die Analyse werden die Daten der zweiten Welle der BAuA-Arbeitszeitbefragung aus dem Jahr 2017 herangezogen (für eine detaillierte Beschreibung der Stichprobe s. Häring et al. 2018). Die Erhebung ist eine repräsentative deutsche Erwerbstätigenbefragung, die im Paneldesign (Längsschnitt) durchgeführt wird und 2015 zum ersten Mal erhoben wurde. Bei der zweiten Befragung 2017 wurde erstmalig nach verkürzten Ruhezeiten gefragt. Die Daten umfassen insgesamt 10.459 Personen (darunter 7446 Panelfälle). Für die Analysen wird die Stichprobe auf abhängig Beschäftigte zwischen 18 und 65 Jahren beschränkt, die einer Vollzeitbeschäftigung von mindestens 35 Stunden pro Woche nachgehen $(n=6753)$. Für die Pfadanalyse werden Personen ausgeschlossen, die fehlende Werte bei den Prädiktoren der Regressionen aufweisen, daher reduziert sich die Stichprobe auf $n=6136$ für die Analysen in den Model$\operatorname{len}^{2} .63 \%$ der Befragten sind männlich, der Altersschnitt liegt bei 48 Jahren $(S D=10,16$ Jahre). Acht Prozent sind in Schichtarbeit beschäftigt. Eine deskriptive Darstellung der Zusammensetzung der Stichprobe findet sich im Anhang (Tab. A.1).

\subsection{Unabhängige Variablen bzw. Mediatorvariablen}

Verkürzte Ruhezeiten Verkürzte Ruhezeiten werden über eine Selbstauskunft erfasst. Als Referenzrahmen für verkürzte Ruhezeiten dient die Mindestruhezeit des Arbeits-

\footnotetext{
${ }^{2}$ Die Abweichungen beruhen hauptsächlich auf Personen, die keine vertraglich vereinbarte Arbeitszeit angegeben haben. Für diese Personen kann keine Überstundenzahl berechnet werden.
} 
zeitgesetzes von elf Stunden ( $\$ 5$ ArbZG). Die Frage hierzu lautet: „Kommt es bei Ihnen - wenn auch nur gelegentlich - vor, dass weniger als elf Stunden zwischen dem Ende eines Arbeitstages und dem Start des nächsten Arbeitstages liegen?“. Die Befragten können mit ,ja“ oder „nein“ antworten. Bei Zustimmung wird nach der Häufigkeit gefragt (,Wie viele Male im Monat ist das normalerweise der Fall?"“). Für die folgenden Analysen wurde eine dichotome Variable auf Basis der beiden Fragen gebildet. Die Variable unterscheidet zwischen Befragten, die mindestens einmal im Monat verkürzte Ruhezeiten aufweisen und Befragten bei denen verkürzte Ruhezeiten seltener als einmal im Monat oder gar nicht auftreten. Dabei können Personen mit gesetzlichen, tarifvertraglichen oder betrieblichen Ausnahmeregelungen nicht identifiziert werden.

Durchschnittliche Tagesarbeitszeit In der BAuA-Arbeitszeitbefragung wird zunächst nach der tatsächlichen Wochenarbeitszeit in Stunden gefragt (,Wie viele Stunden arbeiten Sie normalerweise im Durchschnitt pro Woche in Ihrer Haupttätigkeit - einschließlich regelmäßig geleisteter Überstunden, Mehrarbeit, Bereitschaftsdienst usw.?") Die Frage wird offen gestellt und kann metrisch beantwortet werden, zudem gibt es die Ausweichkategorie ,unregelmäßige Arbeitszeiten“, die in den Analysen jedoch nicht berücksichtigt wurde (fehlender Wert). Zur Normierung auf die Tagesarbeitszeit wurde die tatsächliche Wochenarbeitszeit durch die Anzahl der Arbeitstage pro Woche (Frage: „Wie viele Tage pro Woche arbeiten Sie normalerweise?“) dividiert. Für eine bessere Interpretierbarkeit der Ergebnisse wurde von der so berechneten Tagesarbeitszeit für die inferenzstatistischen Analysen zusätzlich der Wert 8 entsprechend der üblichen werktäglichen Höchstarbeitszeit nach $\S 3$ ArbZG - abgezogen.

Überstunden pro Tag Neben der tatsächlichen wird auch die vertraglich vereinbarte Wochenarbeitszeit in Stunden erfasst („Wie viele Wochenstunden beträgt in Ihrer Tätigkeit Ihre mit dem Arbeitgeber vertraglich vereinbarte Wochenarbeitszeit ohne Überstunden?"). Zur Errechnung der Überstunden wurde für alle Befragten, die eine vertragliche und tatsächliche Wochenarbeitszeit angegeben hatten, jeweils die Differenz zwischen der tatsächlichen und der vertraglich vereinbarten Wochenarbeitszeit errechnet. Überstunden ergeben sich aus der positiven Differenz zwischen tatsächlicher und vertraglicher Wochenarbeitszeit. Um auf das Tagesniveau zu normieren wurde die Variable analog zur tatsächlichen Tagesarbeitszeit durch die Anzahl der Arbeitstage pro Woche geteilt.

Überstunden (Residuen) Lange Arbeitszeiten gehen häufig mit Überstunden einher, d. h. beide Variablen sind hoch miteinander korreliert. Um den inkrementellen Einfluss von
Überstunden auf die abhängigen Variablen bemessen zu können, wurde eine lineare Regression der tatsächlichen Tagesarbeitszeit auf die Überstunden pro Tag berechnet. Die Residuen (Differenz des durch das Regressionsmodell vorhergesagten Werts und dem tatsächlichen Wert: $\hat{y}-y$ ) dieses bivariaten Regressionsmodells geben für jede Person den Anteil der Überstunden an, der nicht durch die Länge der tatsächlichen Tagesarbeitszeit erklärt wird. Durch die Aufnahme der Residuen in das Gesamtmodell wird der Einfluss von Überstunden herausgerechnet (partielle Regression). Partielle Regressionen führen zu einer Reduktion inhaltlich logischer, hoher Kovarianzen zwischen Prädiktoren in Regressionsanalysen (Greene 2003, S. 28).

\subsection{Abhängige Variablen}

Psychosomatische Beschwerden In der BAuA-Arbeitszeitbefragung 2017 werden verschiedene psychosomatische Beschwerden der Beschäftigten erfragt (,Geben Sie bitte an, ob die folgenden Beschwerden bei Ihnen in den letzten 12 Monaten (während der Arbeit bzw. an Arbeitstagen) häufig aufgetreten sind“). In Anlehnung an Franke (2015) und Müller et al. (2018) wurden die folgenden elf Beschwerden berücksichtigt: Rücken- und Kreuzschmerzen, Nacken- und Schulterschmerzen, Kopfschmerzen, Schlafstörungen, Müdigkeit und Erschöpfung, Magen- und Verdauungsbeschwerden, Hörverschlechterung und Ohrgeräusche, Nervosität und Reizbarkeit, Niedergeschlagenheit, körperliche Erschöpfung sowie emotionale Erschöpfung. Die Befragten werden gebeten anzugeben, ob die jeweiligen Beschwerden bei ihnen auftreten (,ja“/,nein“). Für die Analysen wurde die Summe aller psychosomatischer Beschwerden gebildet (Anzahl der ,ja“-Antworten).

Work-Life-Balance Die Operationalisierung der Work-LifeBalance erfolgte über das Einzelitem „Es gelingt mir, bei der Arbeitszeitplanung auf meine familiären und privaten Interessen Rücksicht zu nehmen.“ Die Antwort wird mit Hilfe einer fünfstufigen endpunktbenannten Likert-Skala von 1 ,trifft überhaupt nicht zu“ bis 5 ,trifft voll und ganz zu“" gegeben.

\subsection{Kontrollvariablen}

In den multivariaten Analysen wurde jeweils für das Alter (in Jahren), ob Kinder unter 18 Jahren im Haushalt leben sowie den Beruf und die Tätigkeit der Befragten kontrolliert. Um die Tätigkeitsinhalte der Befragten kontrollieren zu können, wurde zwischen geistiger bzw. körperlicher Tätigkeit unterschieden. Die Frage hierzu lautet: „Sind Sie bei Ihrer Arbeit vorwiegend geistig, vorwiegend körperlich oder etwa gleichermaßen geistig und körperlich tätig?" Die 
Antwortmöglichkeit ist dreifach gestuft (,,geistig“", ,körperlich“, ,gleichermaßen geistig und körperlich“).

Für den beruflichen Tätigkeitsbezug wurde das Anforderungsniveau nach der Klassifikation der Berufe 2010 genutzt (KldB 2010, vgl. Bundesagentur für Arbeit 2015). Die Berufe werden bei der Befragung offen abgefragt und anschließend anhand der KldB 2010 kodiert. Jedem Beruf der KldB 2010 ist ein Anforderungsniveau zugeordnet, welches die Komplexität des jeweiligen Berufes abbildet. Hierbei werden vier Anforderungsniveaus unterschieden: Helfer- und Anlerntätigkeiten, fachlich ausgerichtete Tätigkeiten, komplexe Spezialistentätigkeiten und hoch komplexe Tätigkeiten. Für die inferenzstatistischen Analysen wurden Dummy-Variablen für die verschiedenen Ausprägungen dieser ordinalen Variablen erstellt. Als Referenzkategorien dienen die Kategorien ,gleichermaßen geistig und körperlich“ und die Gruppe der „Helfer- und Anlerntätigkeiten“.

\subsection{Design und Datenanalyse}

Zur Beantwortung der Fragestellungen wurden Pfadmodelle mit Mplus Version 7.4 (Muthén und Muthén 2015) mittels eines Maximum Likelihood Schätzers spezifiziert. Da sich sowohl psychosomatische Beschwerden und die WorkLife-Balance als auch weitere unbeobachtete Anforderungen von Männern und Frauen stark unterscheiden, wurden alle Modelle für die Gesamtstichprobe sowie stratifiziert nach Geschlecht berechnet. Zudem wurde eine Stratifizierung nach Schichtarbeit vorgenommen, wobei Schichtarbeit hier Arbeit in Wechselschicht und Nachtarbeit umfasst.

Um zu schätzen, welcher Anteil der Varianz durch die Kontrollvariablen aufgeklärt wird, wurde zunächst ein sogenanntes Nullmodell erstellt, das die alleinigen Zusammenhänge zwischen den Kontrollvariablen und verkürzten $\mathrm{Ru}$ hezeiten sowie der Work-Life-Balance modelliert. Im zweiten Schritt wurde ein sogenanntes Haupteffektmodell berechnet, das die Zusammenhänge der täglichen Arbeitszeit, der täglichen Überstunden und der verkürzten Ruhezeit mit der Work-Life-Balance sowie den psychosomatischen Beschwerden beinhaltet. Beide Modelle werden als Grundlage für einen Modellvergleich für die folgenden Forschungsmodelle herangezogen.

Als erstes Forschungsmodell wurde ein Mediationsmodell berechnet, das die direkten Effekte der täglichen Arbeitszeit und der täglichen Überstunden (Residuen) auf die Work-Life-Balance bzw. die psychosomatischen Beschwerden sowie die indirekten Effekte, vermittelt über verkürzte Ruhezeiten (Mediator), schätzt (vgl. Abb. 1). Darüber hinaus wurde ein Interaktionsmodell aufgestellt, das sowohl die Haupteffekte der täglichen Arbeitszeit, täglichen Überstunden (Residuen) und verkürzten Ruhezeiten auf die Work-Life-Balance beinhaltet als auch die Interaktion zwi- schen täglicher Arbeitszeit und verkürzten Ruhezeiten sowie die Interaktion zwischen täglichen Überstunden (Residuen) und verkürzten Ruhezeiten (vgl. Abb. 2).

In beiden Modellen wurde für die oben genannten Kontrollvariablen kontrolliert. Außerdem wurden standardisierte und unstandardisierte Koeffizienten, der Anteil der aufgeklärten Varianz $\left(R^{2}\right)$ für die Work-Life-Balance bzw. die psychosomatischen Beschwerden sowie die verkürzten Ruhzeiten berechnet. Als Maße der Modellgüte wurde das Akaike Informationskriterium (AIC) sowie das Bayesianische Informationskriterium $(B I C)$ herangezogen. Geschlechterunterschiede und Abweichungen zwischen Schichtarbeitenden und Beschäftigten ohne Schichtarbeit wurden mit Z-Tests (nach Clogg et al. 1995) untersucht. Ergebnisse zu den Geschlechterunterschieden und Unterschieden zwischen Beschäftigten mit und ohne Schichtarbeit werden nur berichtet, wenn signifikante Ergebnisse vorliegen.

\section{Ergebnisse}

Die Ergebnisse des Haupteffekt-, Mediations- und Interaktionsmodells können den Abbildungen (s. Abb. 3, 4 und 5) entnommen werden. Eine Korrelationsmatrix (Tab. A.2) sowie detaillierte Darstellung der Modelle inklusive Kontrollvariablen und standardisierter Beta-Koeffizienten (Tab. A.3 bis A.6) finden sich im Anhang. Dort ist auch die Stratifizierung nach Geschlecht und Schichtarbeit dargestellt. Das Nullmodell wird nur für den Vergleich benötigt und ist daher ausschließlich in Tabelle A.3 im Anhang zu finden. Die Ergebnisse der Modellvergleiche ( $F$-Test) sind zusätzlich in Tabelle A.7 im Anhang dargestellt.

\subsection{Deskriptive Befunde}

Rund $20 \%$ der Vollzeitbeschäftigten $(n=1172)$ geben an, im Schnitt mindestens einmal monatlich von verkürzten Ruhezeiten $(<11 \mathrm{~h})$ betroffen $\mathrm{zu}$ sein. Frauen und Männer unterscheiden sich nicht nennenswert voneinander ( $21 \%$ vs. $18 \%$ ). Bei den Schichtarbeitenden treten verkürzte Ruhezeiten etwas häufiger auf als bei Beschäftigten ohne Schichtarbeit (26\% vs. 19\%). Insgesamt berichten Beschäftigte mit verkürzten Ruhezeiten etwas mehr psychosomatische Beschwerden $(M=4,1 ; S D=3,03)$ als Beschäftigte ohne verkürzte Ruhezeiten $(M=3,5 ; S D=2,84)$. Auch die Work-Life-Balance wird bei verkürzten Ruhezeiten schlechter bewertet $(M=3,3 ; S D=1,12)$ als ohne verkürzte Ruhezeiten $(M=3,7 ; S D=1,07)$. Mit verkürzten Ruhezeiten gehen längere Tagesarbeitszeiten und mehr Überstunden pro Tag einher (vgl. Tab. A.1). 
Abb. 3 Ergebnisdarstellung Haupteffektmodell (Standardisierte Pfad- bzw. Korrelationskoeffizienten), $* p<0,05$; $* * p<0,01$

Fig. 3 Results main effect model (standardized path and correlation coefficients) $* p<0,05 ; * * p<0,01$

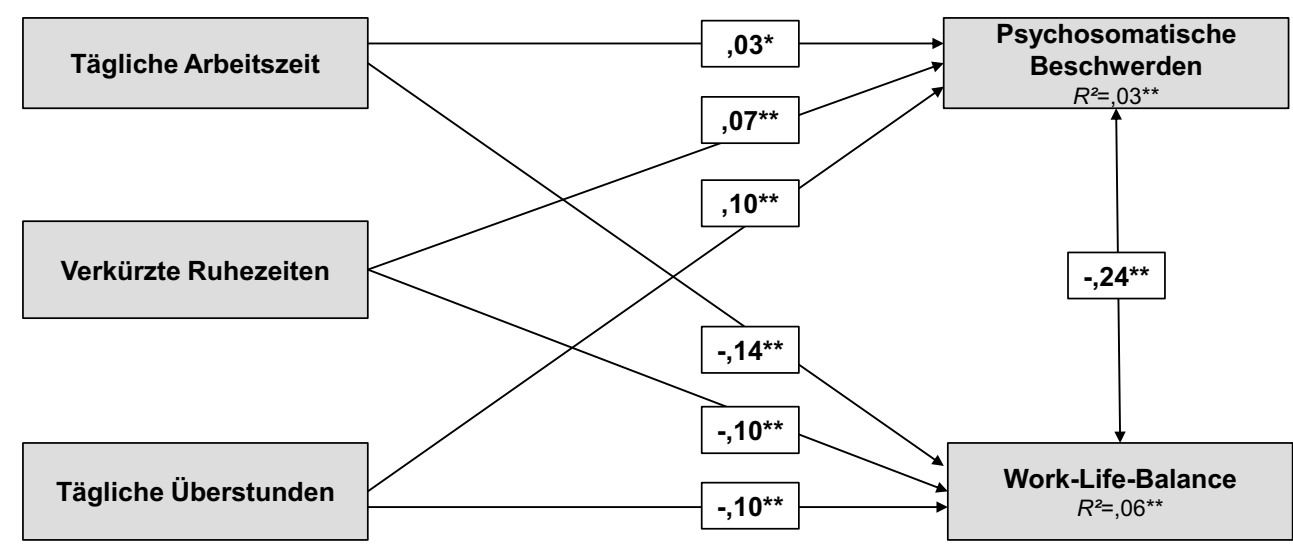

\subsection{Statistische Modellschätzungen}

Haupteffektmodell Das Haupteffektmodell (vgl. Abb. 3) zeigt einen signifikant positiven Zusammenhang zwischen der täglichen Arbeitszeit $(\beta=0,03)$, der täglichen Überstunden $(\beta=0,10)$ sowie der verkürzten Ruhezeiten $(\beta=0,07)$ mit der Anzahl psychosomatischer Beschwerden. Der Zusammenhang zwischen der täglichen Arbeitszeit $(\beta=-0,14)$, den täglichen Überstunden $(\beta=-0,10)$ sowie verkürzter Ruhezeiten $(\beta=-0,10)$ mit der Work-Life Balance ist hingegen statistisch signifikant negativ.

Die stratifizierten Modelle zeigen, dass der Zusammenhang zwischen verkürzten Ruhezeiten und der Anzahl psychosomatischer Beschwerden wie auch der Work-Life-Balance bei Frauen etwas größer ist als bei Männern. Deutlich größer ist auch der Zusammenhang zwischen verkürzten Ruhezeiten und der Anzahl psychosomatischer Beschwer- den bei Schichtarbeitenden im Vergleich zu Beschäftigten ohne Schichtarbeit. Ein statistisch signifikanter Zusammenhang zwischen verkürzten Ruhezeiten und der Work-LifeBalance ist jedoch nur für Beschäftigte ohne Schichtarbeit festzustellen.

Mediationsmodell Im Mediationsmodell (Abb. 4) zeigt sich zunächst ein signifikanter positiver Zusammenhang zwischen täglichen Arbeitszeiten und verkürzten Ruhezeiten $(\beta=0,23)$ sowie zwischen täglichen Überstunden und verkürzten Ruhezeiten $(\beta=0,18)$. Zudem werden signifikant positive direkte Effekte der täglichen Arbeitszeit $(\beta=0,03)$ und täglicher Überstunden $(\beta=0,10)$ auf psychosomatische Beschwerden deutlich. Im Modell sind darüber hinaus die indirekten ${ }^{3}$ Effekte über den Mediator verkürzte Ruhezeiten signifikant positiv (tägliche Arbeitszeit: $\beta=0,02$; tägliche

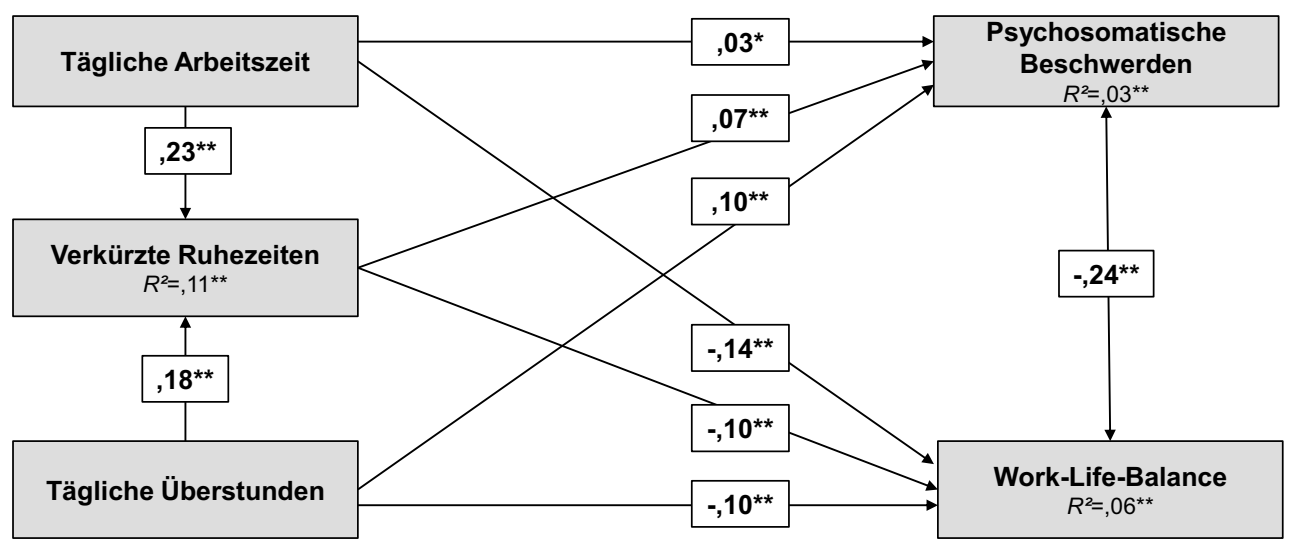

Abb. 4 Ergebnisdarstellung Pfadmodell der Mediation durch verkürzte Ruhezeiten (Standardisierte Pfad- bzw. Korrelationskoeffizienten), $* p<0,05$; ** $p<0,01$. Anmerkung: Der Mediationseffekt entspricht dem Produkt der beteiligten Pfadkoeffizienten. Rechenbeispiel: Mediation verkürzter Ruhezeiten des Effekts täglicher Arbeitszeit auf psychosomatische Beschwerden: $\beta=0,23 * 0,07=0,02$

Fig. 4 Results mediation model (standardized path and correlation coefficients) $* p<0,05 ; * *<0,01$. Note: The mediation effect corresponds to the product of the paths involved. Example: Mediation by quick returns of the effect of daily working time on psychosomatic symptoms: $\beta=0,23$ $* 0,07=0,02$ 
Abb. 5 Ergebnisdarstellung Pfadmodell der Interaktion mit verkürzten Ruhezeiten (Standardisierte Pfad- bzw. Korrelationskoeffizienten), $* p<0,05$; $* * p<0,01$

Fig. 5 Results interaction model (standardized path and correlation coefficients) $* p<0,05 ; * * p<0,01$

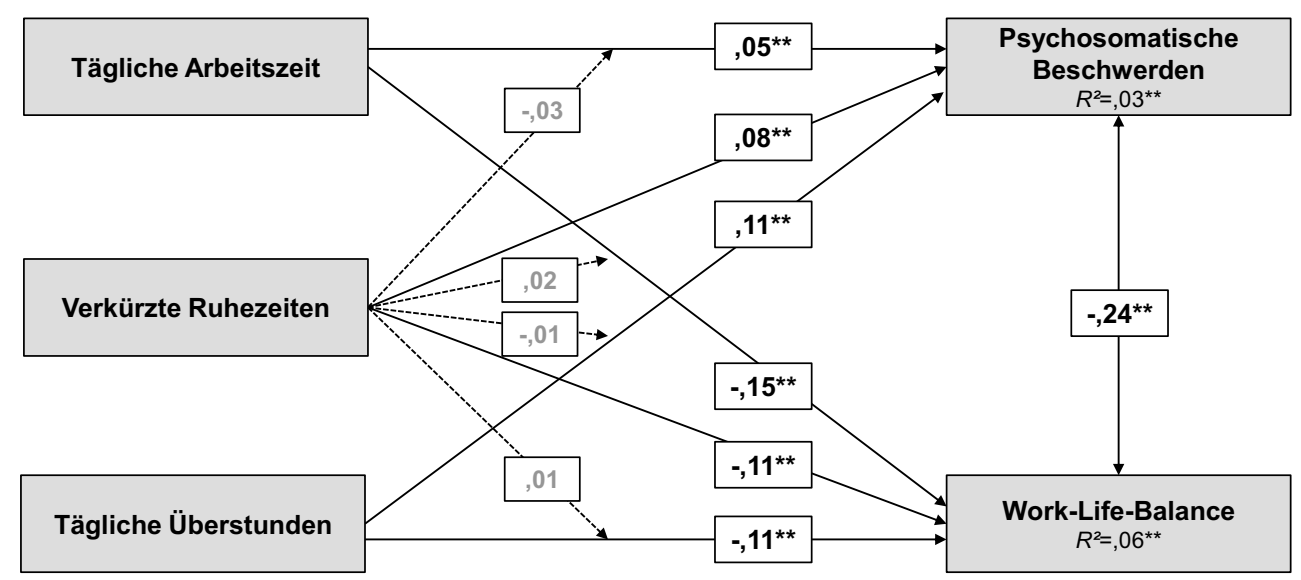

Überstunden $\beta=0,01)$. Der Gesamteffekt ${ }^{4}$ langer Arbeitszeiten auf psychosomatische Beschwerden ist zu ca. $35 \%$ auf verkürzte Ruhezeiten zurückzuführen, der Gesamteffekt von Überstunden zu ca. 11\% (vgl. Tab. 1).

Außerdem zeigen sich signifikant negative direkte Effekte der täglichen Arbeitszeit $(\beta=-0,14)$ und der täglichen Überstunden $(\beta=-0,10)$ auf die Work-Life-Balance. Auch die indirekten Effekte von täglichen Arbeitszeiten $(\beta=-0,02)$, sowie täglichen Überstunden $(\beta=-0,02)$ über den Mediator verkürzte Ruhezeiten auf die Work-Life-Balance sind signifikant negativ. Insgesamt können jeweils $14 \%$ des Gesamteffekts täglicher Arbeitszeiten bzw. 16\% bei den täglichen Überstunden auf die Mediation durch verkürzte Ruhezeiten zurückgeführt werden (vgl. Tab. 1).

Mit Blick auf Geschlechterunterschiede (vgl. Tab. A.8 im Anhang) zeigt sich, dass tägliche Arbeitszeiten die Ruhezeiten bei Männern signifikant stärker vorhersagen als bei Frauen. Das gleiche Bild zeigt sich auch für die täglichen Überstunden. Allerdings sind die direkten Effekte der täglichen Arbeitszeit auf die psychosomatischen Beschwerden bei Frauen stärker zu beobachten.

Im Hinblick auf Unterschiede zwischen Personen mit und ohne Schichtarbeit (vgl. Tab. A.9 im Anhang), zeigt sich, dass verkürzte Ruhezeiten bei Schichtarbeitenden eher mit den Überstunden, bei Personen ohne Schichtarbeit stärker mit der Länge der Arbeitszeit zusammenhängen. Sowohl der direkte als auch der indirekte Effekt von Überstunden auf die Anzahl der psychosomatischen Beschwerden ist bei Schichtarbeitenden stärker ausgeprägt. Bezüglich der Work-Life-Balance hingegen lassen sich keine direkten oder indirekten Effekte für Schichtarbeitende erkennen. Die Effektstärken sind insgesamt deutlich geringer, sodass davon ausgegangen werden kann, dass die nicht-signifikanten Ergebnisse nicht nur auf einer kleineren Stichprobe der Schichtarbeitenden beruhen. Die im Gesamtmodell si-

${ }^{4}$ Der Gesamteffekt ergibt sich aus der Summe von indirektem und direktem Effekt (vgl. Tab. 1). gnifikanten Effekte scheinen sich also primär auf NichtSchichtarbeitende zurückführen zu lassen.

Interaktionsmodell Um zu prüfen, ob die Effekte von verkürzten Ruhezeiten in erster Linie in Kombination mit langen Arbeitszeiten bzw. Überstunden auftreten, wurde darüber hinaus ein Interaktionsmodell berechnet (vgl. Abb. 5). Mit Blick auf die Haupteffekte zeigen sich signifikante Effekte der täglichen Arbeitszeit, der täglichen Überstunden (Residuen) und der verkürzten Ruhezeiten auf psychosomatischen Beschwerden und die Work-Life-Balance von Beschäftigten (vgl. Abb. 5). Weder der Interaktionseffekt zwischen täglicher Arbeitszeit und verkürzten Ruhezeiten noch der Interaktionseffekt zwischen täglichen Überstunden und verkürzten Ruhezeiten hängt signifikant mit den psychosomatischen Beschwerden oder der Work-Life-Balance zusammen. Dies gilt auch für eine stratifizierte Betrachtung nach Geschlecht oder Schichtarbeit.

Güte der Modelle und Modellvergleich Beim Vergleich der Anpassungsgüte $(A I C, B I C)$ zeigt sich eine Überlegenheit des Mediationsmodells gegenüber dem Null- bzw. Haupteffektmodell. Modellvergleiche mittels F-Tests (Tab. A.7) sprechen ebenfalls für eine höhere Varianzaufklärung im Mediations- als im Nullmodell. Dies gilt sowohl für die Modelle der Gesamtstichprobe als auch für die Modelle der Teilstichproben von Männern, Frauen, Erwerbstätige, die Schichtarbeit und keine Schichtarbeit leisten. Das Interaktionsmodell klärt inkrementell mehr Gesamtvarianz auf als das Haupteffektmodell (Tab. A.7) und zeigt zudem eine bessere Anpassung als die anderen Modelle (Tab. 2). Allerdings ist der Zuwachs an Varianzaufklärung im Interaktionsmodell vergleichsweise gering. Zudem zeigen die zusätzlich ins Modell aufgenommenen, entscheidenden Interaktionsterme keine signifikanten Effekte. Insgesamt ergeben sich aus dem Interaktionsmodell daher keine zusätzlichen inhaltlich und statistisch bedeutsamen Zusammenhänge, die über die des Haupteffektmodells hinausgehen. 
Tab. 1 Berechnung der Mediationseffekte (standardisierte $\beta$-Koeffizienten bzw. Anteil der Mediationseffekte am Gesamteffekt in Prozent)

Table 1 Calculation of mediation effects (standardized $\beta$ coefficients and share of mediation effects in total effect in percent)

\begin{tabular}{|c|c|c|c|c|c|c|c|}
\hline \multirow{2}{*}{$\begin{array}{l}\text { Unabhängige } \\
\text { Variable } \\
\text { (UV) }\end{array}$} & \multirow[t]{2}{*}{$\begin{array}{l}\text { Abhängige } \\
\text { Variable (AV) }\end{array}$} & \multirow{2}{*}{$\begin{array}{l}\text { Direkter } \\
\text { Effekt }^{\mathrm{a}} \\
(\mathrm{UV}, \mathrm{AV})\end{array}$} & \multicolumn{3}{|c|}{$\begin{array}{l}\text { Mediationseffekt }{ }^{\mathrm{b}} \text { (indirekter Effekt) durch verkürzte Ru- } \\
\text { hezeiten (RZ) }\end{array}$} & \multirow{2}{*}{$\begin{array}{l}\text { Gesamteffekt }^{\mathrm{c}} \\
\text { (UV, AV und } \\
\text { Mediation) }\end{array}$} & \multirow{2}{*}{$\begin{array}{l}\text { Anteil der } \\
\text { Mediation an } \\
\text { Gesamteffekt }^{\mathrm{d}} \\
\text { in } \%\end{array}$} \\
\hline & & & UV, RZ & $\mathrm{RZ}, \mathrm{AV}$ & Mediation $^{\mathrm{b}}$ & & \\
\hline \multirow{2}{*}{$\begin{array}{l}\text { Tägliche } \\
\text { Arbeitszeit } \\
\text { in Stunden }\end{array}$} & $\begin{array}{l}\text { Psychos. Be- } \\
\text { schwerden }\end{array}$ & 0,03 & 0,23 & 0,07 & 0,02 & 0,05 & 35 \\
\hline & $\begin{array}{l}\text { Work-Life- } \\
\text { Balance }\end{array}$ & $-0,14$ & & $-0,10$ & $-0,02$ & $-0,16$ & 14 \\
\hline \multirow{2}{*}{$\begin{array}{l}\text { Tägl. Über- } \\
\text { stunden } \\
\text { (Residuen) }\end{array}$} & $\begin{array}{l}\text { Psychos. Be- } \\
\text { schwerden }\end{array}$ & 0,10 & 0,18 & 0,07 & 0,01 & 0,12 & 11 \\
\hline & $\begin{array}{l}\text { Work-Life- } \\
\text { Balance }\end{array}$ & $-0,10$ & & $-0,10$ & $-0,02$ & $-0,12$ & 16 \\
\hline
\end{tabular}

${ }^{\mathrm{a}} \beta_{\text {Direkt }}=\beta_{\mathrm{UV}, \mathrm{AV}} ;{ }^{\mathrm{b}} \beta_{\text {Mediation }}=\beta_{\mathrm{UV}, \mathrm{RZ}} * \beta_{\mathrm{RZ}, \mathrm{AV}} ;{ }^{\mathrm{c}} \beta_{\text {Gesamt }}=\beta_{\text {Direkt }}+\beta_{\text {Mediation }} ;{ }^{d} \beta_{\text {Mediation }} / \beta_{\text {Gesamt }}$

Tab. 2 Güteindikatoren zu den Modellen und Stichproben

Table 2 Goodness of fit indices for different models and samples

\begin{tabular}{lllllll}
\hline Modelle & Index & Gesamt & Männer & Frauen & Schichtarbeit & Keine Schichtarbeit \\
\hline Nullmodell & AIC & $53.946,32$ & $33.786,03$ & $19.957,32$ & 4849,37 & $48.599,42$ \\
& BIC & $54.134,54$ & $33.961,22$ & $20.117,84$ & 4967,93 & $48.785,01$ \\
Haupteffektmodell & AIC & $53.617,92$ & $33.614,96$ & $19.818,44$ & 4823,35 & $48.289,77$ \\
& BIC & $53.846,46$ & $33.827,69$ & $20.013,36$ & 4967,32 & $48.515,13$ \\
Mediationsmodell & AIC & $53.112,49$ & $33.226,69$ & $19.695,56$ & 4806,33 & $47.762,22$ \\
& BIC & $53.354,48$ & $33.451,94$ & $19.901,95$ & 4958,76 & $48.000,84$ \\
& AIC & $52.375,31$ & $32.821,57$ & $19.388,33$ & 4700,33 & $47.192,56$ \\
\end{tabular}

AIC Akaike Information Criterion, BIC Bayesian Information Criterion

\section{Diskussion}

\subsection{Interpretation der Befunde}

Einige Studien deuten bereits darauf hin, dass verkürzte $\mathrm{Ru}-$ hezeiten negativ mit sowohl psychosomatischen Beschwerden (Åkerstedt und Kecklund 2017; Dahlgren et al. 2016; Flo et al. 2014; Nielsen et al. 2019; Vedaa et al. 2017a, 2017b) als auch der Work-Life-Balance (z.B. Backhaus et al. 2019; Dahlgren et al. 2016; Karhula et al. 2018, 2017) korreliert sind. Zusammenfassend zeigen auch die Ergebnisse der vorliegenden Studie, dass verkürzte Ruhezeiten (neben langen Arbeitszeiten und Überstunden) ein Risiko für die Gesundheit wie die Work-Life-Balance von Beschäftigten darstellen können. Dabei wurden die Zusammenhänge erstmalig im Rahmen einer großangelegten repräsentativen Erwerbstätigenbefragung untersucht. Bislang fokussierten die meisten Studien verkürzte Ruhezeiten in der Schichtarbeit (Vedaa et al. 2016). Die Ergebnisse zeigen aber, dass die Auswirkungen verkürzter Ruhezeiten nicht nur für Schichtarbeitende von Bedeutung sind, sondern sich auch in der breiten Erwerbsbevölkerung zeigen. Bedingt durch Schichtrotationen, Nachtarbeit und geringen zeitlichen Handlungsspielräumen sind Schichtarbeitende aber stärker belastet als Beschäftigte ohne Schichtarbeit
(Arlinghaus und Lott 2018), was sich auch in psychosomatischen Beschwerden sowie einer schlechteren Work-LifeBalance niederschlägt (Amlinger-Chatterjee 2016; Arlinghaus und Nachreiner 2012; BAuA 2016; Costa 2010; Greubel et al. 2016; Itani und Kaneita 2016; Puttonen et al. 2010; Vogel et al. 2012). Verkürzte Ruhezeiten stellen somit insbesondere für Schichtarbeitende eine zusätzliche Belastung dar. Demensprechend deuten die hier vorliegenden Ergebnisse auch darauf hin, dass der Zusammenhang zwischen verkürzten Ruhezeiten und der Anzahl psychosomatischer Beschwerden für Schichtarbeitende deutlich stärker ausgeprägt ist. Bezüglich der Work-Life-Balance hingegen lassen sich für die Gruppe der Schichtarbeitenden keine signifikante Assoziation mit verkürzten Ruhezeiten erkennen, allerdings wird die Work-Life-Balance von Schichtarbeitenden allgemein schlechter bewertet.

Gleichzeitig ist bekannt, dass psychosomatische Beschwerden und die Work-Life-Balance mit langen $\mathrm{Ar}$ beitszeiten und Überstunden zusammenhängen (BAuA 2016), daher wurden diese bei den vorliegenden Analysen ebenfalls berücksichtigt. Da lange Arbeitszeiten und Überstunden jedoch miteinander korrelieren, wurden die Überstunden in allen Analysen so modelliert, dass lediglich der nicht durch lange Arbeitszeiten erklärte Teil der Überstunden (Residuen) in die Modelle miteinfließt 
(vgl. Kapitel 3.2 Methoden). Der Zusammenhang dieser Überstunden mit Gesundheit ist deutlich größer als der Zusammenhang von täglicher Arbeitszeit mit Gesundheit. Dies deutet darauf hin, dass lange Arbeitszeiten, die durch eine Überschreitung der vereinbarten Arbeitszeiten entstehen, eine andere Qualität darstellen und gesundheitlich relevant sind (BAuA 2016). Obwohl für die Work-Life-Balance sowohl Überstunden als auch lange Arbeitszeiten von großer Bedeutung sind, stellen sich hier die täglichen Arbeitszeiten als nochmals bedeutsamer dar. Vermutlich ist es für die Tagesgestaltung wenig bedeutsam, ob die langen Arbeitszeiten vereinbart sind oder durch Überstunden entstehen, hier steht allgemein die fehlende Freizeit im Vordergrund.

Bezüglich des Zusammenhangs von verkürzten Ruhezeiten und langen Arbeitszeiten bzw. Überstunden erscheint es zunächst nachvollziehbar, dass lange Arbeitszeiten und Überstunden die Wahrscheinlichkeit erhöhen, dass Beschäftigte verkürzte Ruhezeiten aufweisen. Allerdings wird in letzter Zeit auch immer wieder argumentiert, dass viele Beschäftigte ihre Arbeit am Nachmittag unterbrechen wollen, um sich familiären oder privaten Verpflichtungen $\mathrm{zu}$ widmen. Am Abend würden sie ihre Arbeit dann wieder aufnehmen, was eine Unterbrechung der Ruhezeiten hervorrufen kann, aber nicht zwangsläufig zu längeren Arbeitszeiten bzw. kürzeren Gesamtruhezeiten führt (BDA 2015). Entgegen der vorgebrachten Argumentation zeigt der vorliegende Beitrag, dass es einen nennenswerten Zusammenhang zwischen langen Arbeitszeiten sowie Überstunden und verkürzten Ruhezeiten gibt. Das Risiko der Ruhezeitverletzung ist also höher, je längere Tages- bzw. Wochenarbeitszeiten Beschäftigte aufweisen. Dieser Zusammenhang ist bei den Männern deutlicher ausgeprägt als bei Frauen. Schichtarbeitende hingegen scheinen ihre Ruhezeiten häufiger aufgrund von Überstunden zu verletzten. Dies mag darauf zurückzuführen sein, dass sich bei Schichtarbeitenden mit Überstunden das Risiko auf verkürzte Ruhezeiten durch die starre Rotation der Schichten erhöht.

Daran anschließend stellt sich die Frage, inwiefern eine Ruhezeitverkürzung den negativen Zusammenhang zwischen langen Arbeitszeiten sowie Überstunden und der Gesundheit bzw. der Work-Life-Balance zumindest partiell mediiert, oder anders ausgedrückt, inwiefern ein Teil des negativen Effektes langer Arbeitszeiten und Überstunden durch zu kurze Ruhezeiten erklärt werden kann. Pfadmodelle weisen darauf hin, dass verkürzte Ruhezeiten den statistischen Effekt von langen Arbeitszeiten und Überstunden partiell mediieren. Das heißt, dass der negative Zusammenhang zwischen langen Arbeitszeiten sowie Überstunden und psychosomatischen Beschwerden, teilweise auch auf verkürzte Ruhezeiten zurückzuführen ist. Für die Gruppe der Schichtarbeitenden lässt sich der Zusammenhang zwi- schen Überstunden und der Work-Life-Balance hingegen nicht durch verkürzte Ruhezeiten erklären.

\subsection{Kritische Reflexion}

Die vorliegende Studie stellt eine erste Analyse verkürzter Ruhezeiten auf Basis repräsentativer Daten über alle Beschäftigtengruppen in Deutschland dar. Gleichzeitig ist anzumerken, dass die Ergebnisse auf Querschnittsdaten basieren und folglich nicht kausal interpretierbar sind. In weiterführenden Längsschnitt- und Kohortenstudien sollten die Zusammenhänge zwischen verkürzten Ruhezeiten, Gesundheit und Work-Life-Balance weiter untersucht werden. Allerdings ist ebenso darauf hinzuweisen, dass mögliche negative Auswirkungen zum Teil auch unterschätzt sein können. Wenn Beschäftigte aus gesundheitlichen Gründen ihre Arbeitszeit verkürzen oder ihre Erwerbstätigkeit gänzlich aufgeben müssen, so können sie in der Studie nicht berücksichtigt werden. Dadurch wird der Gesundheitszustand in der Befragung systematisch überschätzt (Healthy Worker Effect, vgl. Li und Sung 1999). Ähnliches gilt für die Zusammenhänge mit der Work-Life-Balance. Stellen Beschäftigte fest, dass sie aufgrund ihrer Arbeitszeit (oder zu kurzer Ruhezeiten) nicht in der Lage sind Privates und Berufliches zu vereinbaren, erhöht sich die Wahrscheinlichkeit der Arbeitszeitanpassung, eines Jobwechsels oder der Aufgabe der Erwerbstätigkeit.

Des Weiteren beruhen alle Analysen auf der Selbstauskunft der Beschäftigten. Die Selbstauskunft ist, zunächst im Hinblick auf die Messung von Arbeitszeiten nicht immer fehlerfrei. Zur Lösung dieses methodischen Problems werden in vielen skandinavischen Ländern Registerdaten herangezogen (Härmä et al. 2015), die in diesem Umfang für Deutschland aber nicht zur Verfügung stehen. Eine Alternative sind Tagebuchstudien, die verkürzte Ruhezeiten genauer beleuchten (Vedaa et al. 2017a). Allerdings sind diese Studien häufig mit einem enormen Forschungsaufwand verbunden bzw. datenschutzrechtlich schwierig. Die Berechnung der täglichen Arbeitszeit bzw. der täglichen Überstunden auf Basis der wöchentlichen Angabe mag zu leichten Verzerrungen führen. Auch die Operationalisierung der psychosomatischen Beschwerden basiert auf der subjektiven Auskunft der Befragten und stellt somit eine indirekte Messung der Gesundheit dar. Einige Studien weisen aber darauf hin, dass die subjektive Gesundheitsmessung mit einer objektiven vergleichbar und als zuverlässig im Hinblick auf den allgemeinen Gesundheitszustand gelten kann (Miilunpalo et al. 1997; Wu et al. 2013). Zudem kann es aufgrund der Parametrisierung der psychosomatischen Beschwerden zu Unschärfen in den Ergebnissen kommen. Da davon auszugehen ist, dass psychosomatische Beschwerden mit unterschiedlichen Beanspruchungsformen einhergehen wurde deswegen zumindest näherungsweise für kör- 
perliche und geistige Tätigkeiten kontrolliert. Gerade in tätigkeitsübergreifenden Studien sind Summenindizes aus unterschiedlichen psychosomatischen Beschwerden jedoch weit verbreitet (Franke 2015; Müller et al. 2018). Zur Erkenntnissicherung wäre darüber hinaus eine Replikation der Ergebnisse mit weiteren Gesundheitsparametern durchaus wünschenswert.

Darüberhinausgehend wäre außerdem zu prüfen, inwiefern die negativen Zusammenhänge verkürzter Ruhezeiten mit Gesundheit und Work-Life-Balance, auf mangelnde Erholung bzw. zu kurzen Schlaf zurückzuführen sind. Insbesondere wenn die entstandene Flexibilität fremdbestimmt ist, d.h. durch Betriebe und Arbeitgeber vorgegeben wird, kann dies das Abschalten von der Arbeit in der Ruhezeit erschweren und zu Konflikten bzw. mangelhafter Erholung beitragen (Amlinger-Chatterjee und Wöhrmann 2017; Lott 2018). Aber auch bei selbstbestimmter Flexibilität von Beschäftigten entstehen Potenziale für eine Selbstgefährdung, die mit langfristigen gesundheitlichen Folgen verbunden sein können (Böhne und Breutmann 2012). Schließlich sollten zukünftig unterschiedliche Beschäftigtengruppen näher betrachtet werden. Zwar wurde in den dargestellten Analysen für Tätigkeit und Bildungsniveau kontrolliert - dennoch wäre es für die aktuelle Debatte hilfreich zu wissen, ob es Beschäftigtengruppen gibt, für die verkürzte Ruhezeiten kein bzw. ein geringeres Risiko darstellen.

\subsection{Praktische Relevanz und Gestaltungsempfehlungen}

In der Analyse wurde zwischen Befragten unterschieden, die mindestens einmal pro Monat eine Ruhezeitverkürzung von unter elf Stunden vorweisen. Selbst bei dieser konservativ angesetzten Grenze der Häufigkeit (einmal) und Mindestruhezeit $(11 \mathrm{~h})$ zeigen sich bereits negative Zusammenhänge mit Gesundheit und Work-Life-Balance. Mindestruhezeiten stellen daher ein wichtiges und sinnvolles Instrument des Arbeitsschutzes dar. Ausnahmeregelungen sind kritisch zu bewerten und sollten in die aktuelle Debatte um eine Überarbeitung des Arbeitszeitgesetzes einflieBen. Bei zeitnahem Ausgleich besteht die Möglichkeit einer Kürzung der Mindestruhezeit auf 10 Stunden für bestimmte Einrichtungen ( $\$ 5$ Abs. 2 ArbZG). Dabei sind Berufsgruppen betroffen, die ohnehin schon ungünstige Arbeitszeitbedingungen aufweisen (vgl. Backhaus et al. 2018), wie z.B. Beschäftigte in Krankenhäusern (Pflegeberufe), Gaststätten (Berufe in Gastronomie und Hotelgewerbe) oder Verkehrsbetrieben (Führerinnen und Führer von Verkehrsund Transportmaschinen). Eine tarifvertragliche, betrieblich oder dienstlich vereinbarte Verkürzung der Ruhezeiten um bis zu zwei Stunden (z.B. § 7 Abs. 1 Nr. 3 ArbZG) erscheint besonders diskussionswürdig. Bei der Betrachtung von Mindestruhezeiten sollte berücksichtigt werden, dass - nach Abzug einer empfohlenen Schlafdauer von etwa acht Stunden - nur drei bzw. bei Verkürzung ggf. nur ein bis zwei Stunden Freizeit verbleiben. Zieht man hiervon arbeitsgebundene Zeiten, wie mögliche Pendel- bzw. Wegezeiten ab, verkürzt sich diese Zeit weiter. Aus diesem Grund bestehen in der Schlafforschung seit langem Forderungen, die Mindestruhezeit auszuweiten, um eine ausreichende Schlafdauer und Erholung sowie ausreichende freie Zeit für private Aktivitäten sicherstellen zu können (Kecklund und Åkerstedt 1995).

Ungeachtet dessen gibt es auf Bundes- und Länderebene politische Diskussionen zu einer flexibleren Handhabung der Mindestruhezeiten (Deutscher Bundestag 2018; Landtag Nordrhein-Westfalen 2018). Auch wenn diese Forderungen in erster Linie auf Wissensarbeiterinnen und -arbeiter abzielen, die keine schwere körperliche Arbeit leisten und flexibel von Zuhause aus arbeiten können, sind diese Bestrebungen kritisch zu betrachten. Der vorliegende Beitrag zeigt, dass eine Verkürzung der Ruhezeiten auch unabhängig von der beruflichen Tätigkeit negativ mit Gesundheit und Work-Life-Balance zusammenhängt. Dies ist darauf zurückzuführen, das zum einen Erholung unabhängig von der Tätigkeit, von großer Bedeutung bei der Rückstellung mentaler bzw. kognitiver Beanspruchungsfolgen ist (Arlinghaus 2017). Möglicherweise aber auch darauf, dass die Mindestruhezeit bei wissensintensiven Berufen durch die Erreichbarkeit bzw. Kontaktierung außerhalb der Arbeitszeit extrem verletzt wird (Pauls et al. 2019). Hiervon können Beschäftigte mit einer ohnehin hohen Arbeitsintensität betroffen sein (Hassler et al. 2016). Auch von einer „freiwilligen“ Verletzung der Ruhezeiten sollte eher abgesehen werden, denn Studien weisen darauf hin, dass Freiwilligkeit durch sozialen Druck zur impliziten Vorschrift werden kann (Pangert et al. 2016).

Deshalb sollte für die betriebliche Gestaltung und Bewertung von Arbeitszeiten die Ruhezeit eine wichtige Rolle spielen. Neben einer Berücksichtigung der gesicherten arbeitswissenschaftlichen Erkenntnisse zur Länge, Lage und Flexibilität der Arbeitszeit (Rothe et al. 2017) ist zu empfehlen, auch der Länge der Ruhezeit stärkere Beachtung zu gewähren. Eine Unterschreitung der Mindestruhezeit ist möglichst zu vermeiden und bei der Planung der Arbeitszeit auszuschließen, um eine ausreichende Erholung der Beschäftigten sicherzustellen. Dies gilt insbesondere bei Schichtarbeit mit Wechselschichten, hier liegt umfangreiches Gestaltungswissen zu Ruhezeiten vor (Vedaa et al. 2016).

\subsection{Zusammenfassung und Fazit}

Ruhezeiten dienen der Rückstellung von Beanspruchungsfolgen die während eines Arbeitseinsatzes aufgetreten sind, d.h. der Erholung von der Arbeit. Unabhängig von der Tä- 
tigkeit ist jede bzw. jeder fünfte Vollzeitbeschäftigte von verkürzten Ruhezeiten betroffen. Die statistischen Modelle zeigen, dass verkürzte Ruhezeiten häufig mit langen täglichen Arbeitszeiten, aber auch mit Überstunden einhergehen. Im Vergleich zu Frauen sind die Zusammenhänge bei Männern größer. Verkürzte Ruhezeiten bei Schichtarbeitenden sind häufig auf Überstunden zurückzuführen. Die tägliche Arbeitszeit, Überstunden und verkürzte Ruhezeiten wirken sich negativ auf die Gesundheit (Anzahl psychosomatischer Beschwerden) und die Work-Life-Balance aus. Verkürzte Ruhezeiten mediieren dabei einen signifikanten Anteil des Einflusses, sowohl der täglichen Arbeitszeit als auch der Überstunden. Das Zusammenwirken von verkürzten Ruhezeiten mit täglicher Arbeitszeit bzw. Überstunden (Interaktionsmodell) hat keinen signifikanten Effekt. Es zeigt sich, dass die Verkürzung der Ruhezeiten insgesamt negativ zu beurteilen ist. Mindestruhezeiten stellen folglich zentrale und wirksame Arbeitsschutzstandards dar. Eine Absenkung dieses Mindeststandards, wie er mo- mentan in der arbeitszeitpolitischen Debatte von einigen gefordert wird, ist aus arbeitswissenschaftlicher Sicht nicht empfehlenswert.

Open Access Dieser Artikel wird unter der Creative Commons Namensnennung 4.0 International Lizenz veröffentlicht, welche die Nutzung, Vervielfältigung, Bearbeitung, Verbreitung und Wiedergabe in jeglichem Medium und Format erlaubt, sofern Sie den/die ursprünglichen Autor(en) und die Quelle ordnungsgemäß nennen, einen Link zur Creative Commons Lizenz beifügen und angeben, ob Änderungen vorgenommen wurden.

Die in diesem Artikel enthaltenen Bilder und sonstiges Drittmaterial unterliegen ebenfalls der genannten Creative Commons Lizenz, sofern sich aus der Abbildungslegende nichts anderes ergibt. Sofern das betreffende Material nicht unter der genannten Creative Commons Lizenz steht und die betreffende Handlung nicht nach gesetzlichen Vorschriften erlaubt ist, ist für die oben aufgeführten Weiterverwendungen des Materials die Einwilligung des jeweiligen Rechteinhabers einzuholen.

Weitere Details zur Lizenz entnehmen Sie bitte der Lizenzinformation auf http://creativecommons.org/licenses/by/4.0/deed.de. 


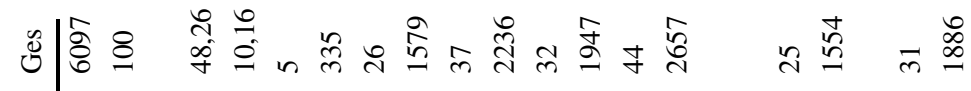

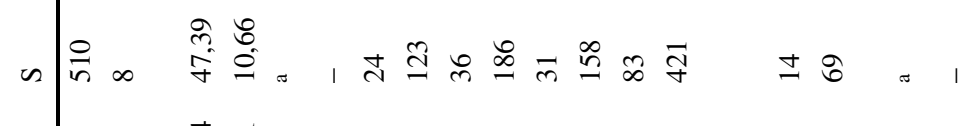

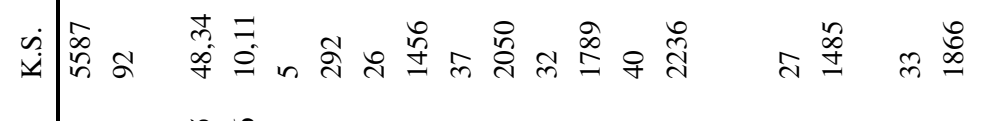

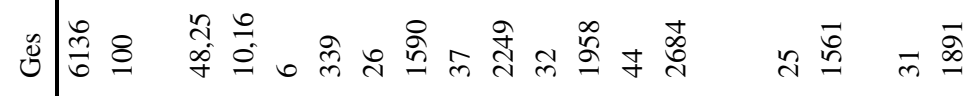

古

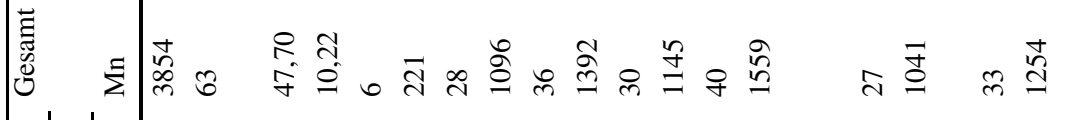

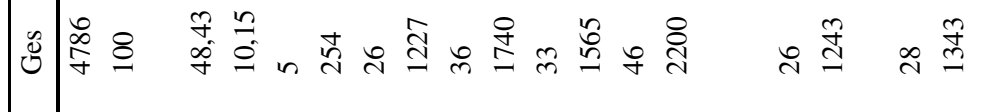

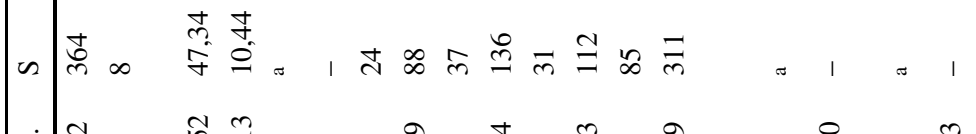

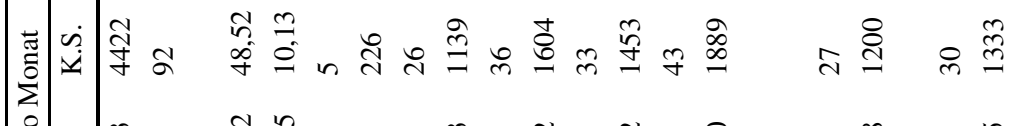

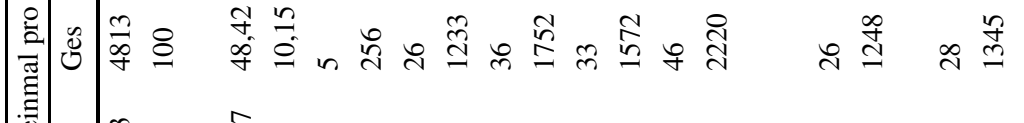

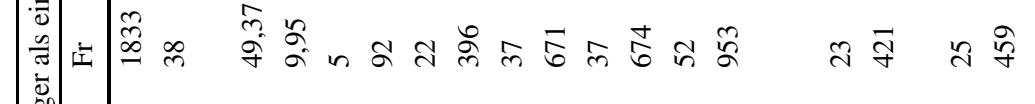

离

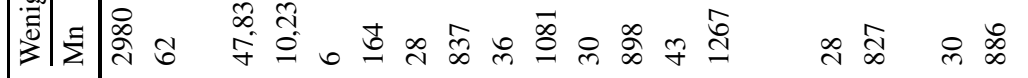

.

.

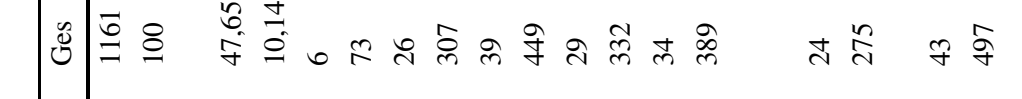

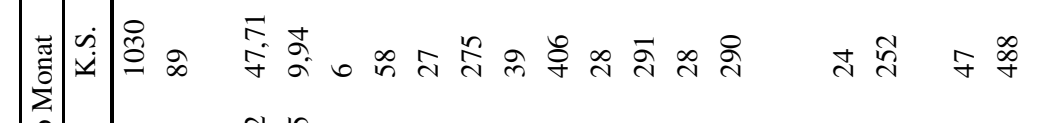




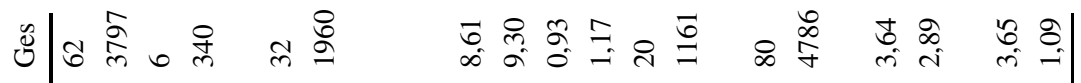

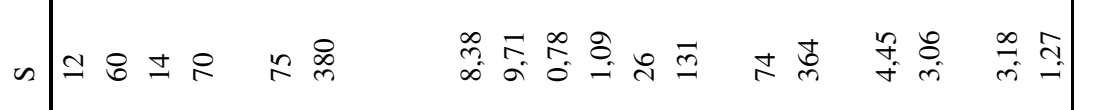

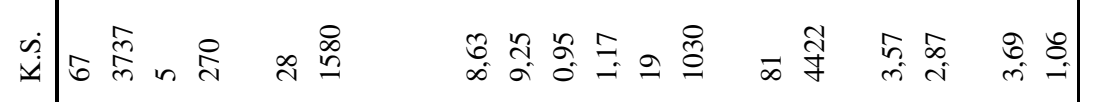

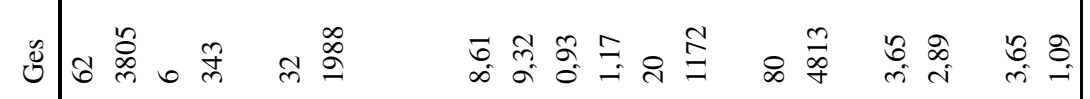

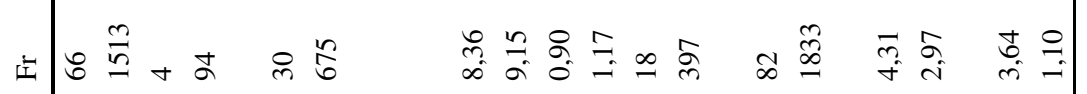

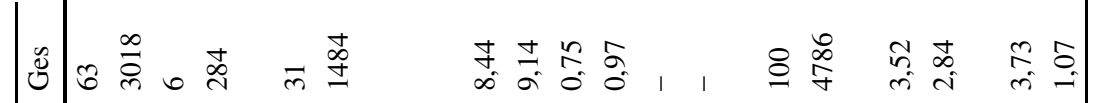

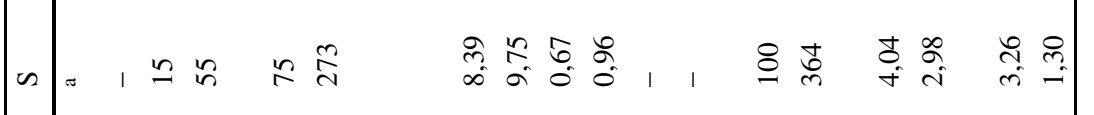

䓂

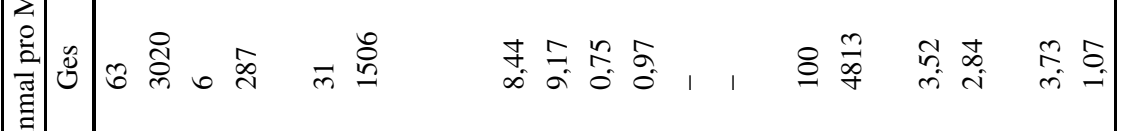

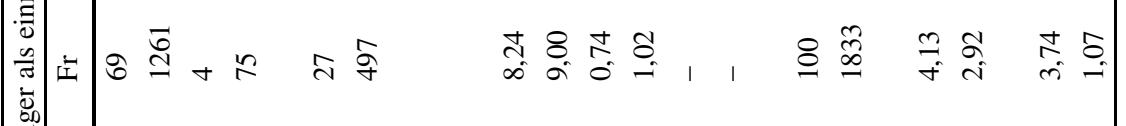

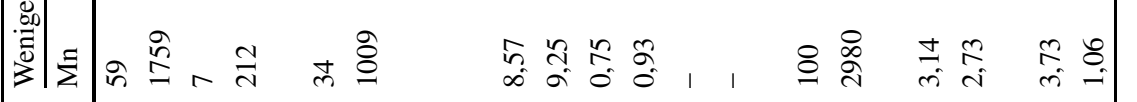

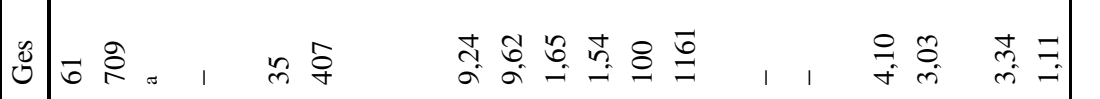

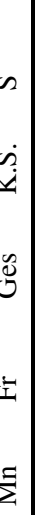

$-1=1 \approx n$

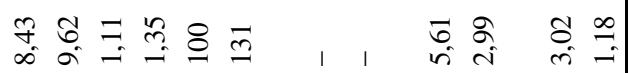

ธิ

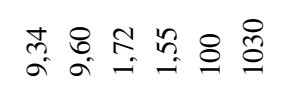

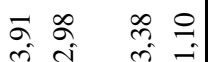

远

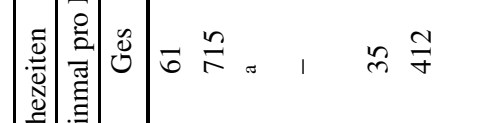

तु่

ले

क्ष

त.

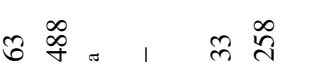

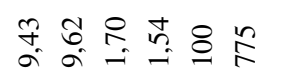

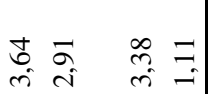

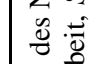

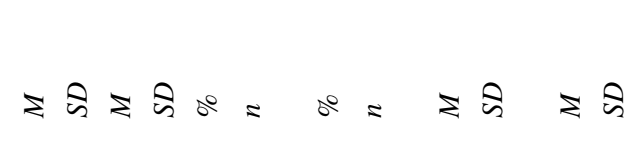

$\therefore=0^{\circ}=0^{\circ}=$

竞

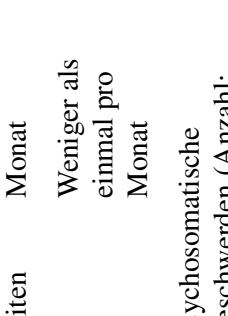




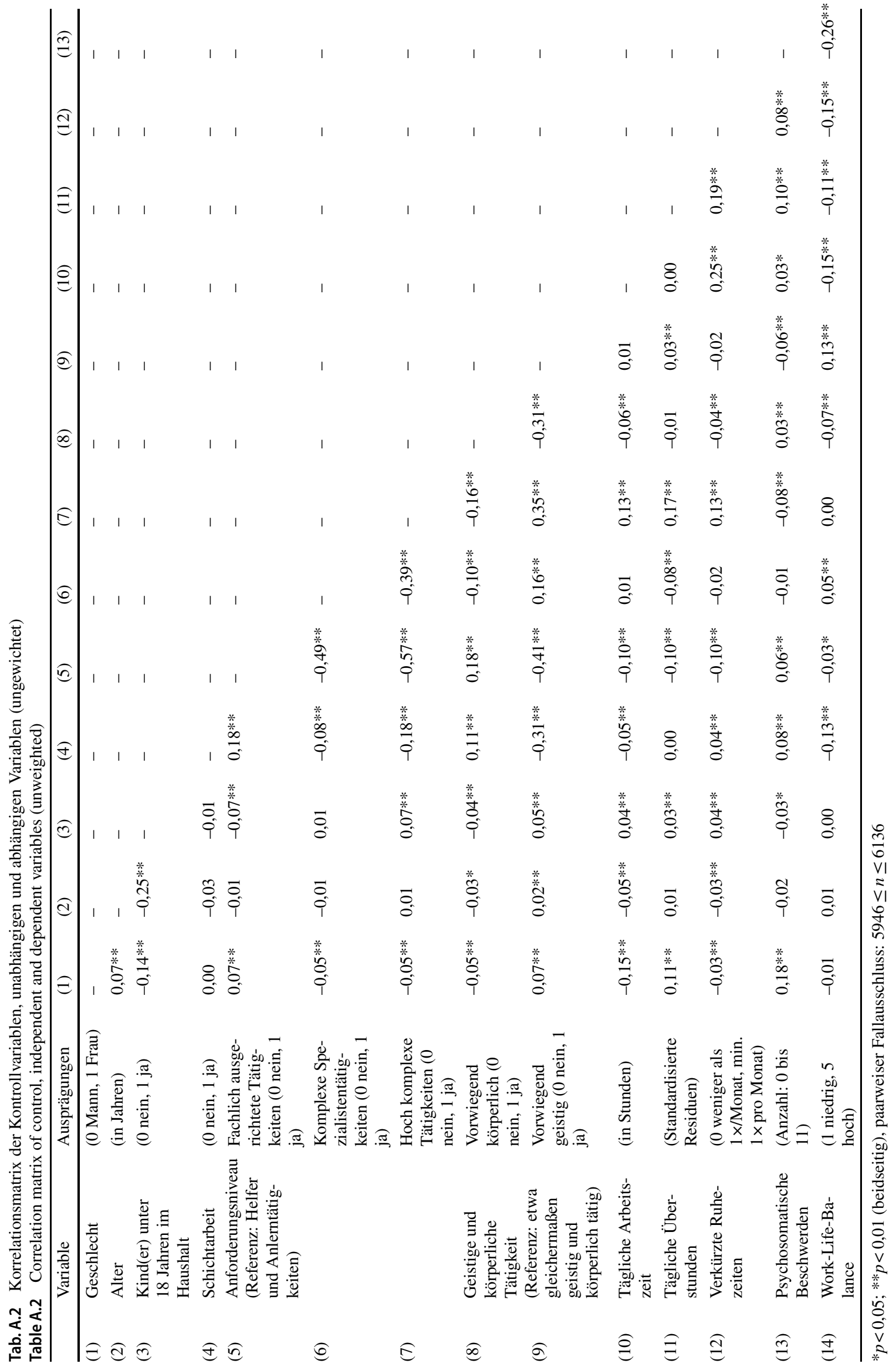


Tab. A.4 Ergebnisse des Haupteffektmodells

Table A.4 Results of the main effect model

Gesamt $(\beta) \quad$ Männer $(\beta) \quad$ Frauen $(\beta) \quad$ Schichtarbeit $(\beta) \quad$ Keine Schichtarbeit $(\beta)$

AV: Verkürzte Ruhezeiten

Alter (in Jahren)

Kind unter 18 im Haushalt

$-0,02$

$-0,01$

$-0,02$

Anforderungsniveau - Referenz:

Helfer- und Anlerntätigkeit

Fachlich ausgerichtete Tätigkeiten

Komplexe Spezialistentätigkeiten

Hoch komplexe Tätigkeiten

0,03

0,03

$-0,03$

$-0,00$

0,03

Art der Tätigkeit - Referenz: gleichermaßen geistig und körperlich

$\begin{array}{lllll}0,05 & 0,01 & 0,13 * & 0,09 & 0,06 \\ 0,10 * * & 0,05 & 0,17 * * & 0,12 & 0,12 * \\ 0,22 * * & 0,16 * * & 0,29 * * & 0,12 * & 0,25 * *\end{array}$

Vorwiegend körperlich

Vorwiegend geistig

$R^{2}$

\section{AV: Psychosomatische Beschwerden}

Alter (in Jahren)

Kind unter 18 im Haushalt

Anforderungsniveau - Referenz:

Helfer- und Anlerntätigkeit

Fachlich ausgerichtete Tätigkeiten

Komplexe Spezialistentätigkeiten

Hoch komplexe Tätigkeiten

Art der Tätigkeit - Referenz:

gleichermaßen geistig und körperlich

Vorwiegend körperlich

Vorwiegend geistig

Tägliche Arbeitszeit

Tägliche Überstunden (Res.)

Verkürzte Ruhezeiten

$R^{2}$

\section{AV: Work-Life-Balance}

Alter (in Jahren)

Kind unter 18 im Haushalt

Anforderungsniveau - Referenz:

Helfer- und Anlerntätigkeit

Fachlich ausgerichtete Tätigkeiten

Komplexe Spezialistentätigkeiten

Hoch komplexe Tätigkeiten
$-0,03 *$

$-0,10 * *$

$-0,04 *$

$0,03 * *$

$0,06 * *$

$0,02 * *$

$-0,02$

$-0,03 *$

$-0,01$

$-0,00$

$-0,12$ **

$-0,14 * *$

$-0,02$

$-0,22 * *$

$-0,05$

$-0,12$ *

0,01

$-0,01$

0,03 *

0,10 **

$0,07 * *$

$0,03 * *$

0,00

0,01

0,06

0,07

0,06

$\begin{array}{ll}-0,04 * * & -0,04 * \\ 0,11 * * & 0,13 * * \\ -0,14 * * & -0,14 * * \\ -0,10 * * & -0,09 * * \\ -0,10^{* *} & -0,09 * * \\ 0,06 * * & 0,05 * * \\ -0,24 * * & -0,21 * *\end{array}$

$-0,05^{*}$

0,09 **

$-0,14 * *$

$-0,10$ **

$-0,12$ **

0,07 **

$-0,29 * *$
$-0,03$

0,06

0,02

$-0,03 *$

$-0,09$ **

0,03 **

$-0,03$

$-0,03$ *

$-0,03$

$-0,08$

$-0,10$

$-0,13$ *

$-0,20$ **

$-0,05$

0,01

0,01

0,01

$0,03 *$

0,10 **

0,05 **

0,02 **

0,00

0,00

0,08

0,05

$-0,02$

$-0,01$

0,00

$-0,02$

gleichermaßen geistig und körperlich

Vorwiegend körperlich

Tägliche Arbeitszeit

Tägliche Überstunden (Res.)

Verkürzte Ruhezeiten

$R^{2}$

Korrelation Work-Life-Balan-

ce $\times$ Psychosomatische Beschwerden

Res Residuen

$* p<0,05 ; * * p<0,01$ 
Tab. A.5 Ergebnisse des Mediationsmodells

Table A.5 Results of the mediation model

Gesamt $(\beta) \quad$ Männer $(\beta) \quad$ Frauen $(\beta) \quad$ Schichtarbeit $(\beta) \quad$ Keine Schichtarbeit

$(\beta)$

AV: Verkürzte Ruhezeiten

Alter (in Jahren)

Kind unter 18 im Haushalt

$-0,01$

$-0,03$

$-0,02$

$-0,02$

Anforderungsniveau - Referenz:

Helfer- und Anlerntätigkeit

Fachlich ausgerichtete Tätigkeiten

Komplexe Spezialistentätigkeiten

Hoch komplexe Tätigkeiten

0,02

0,02

0,01

$-0,01$

0,02

Art der Tätigkeit - Referenz

gleichermaßen geistig und körperlich

Vorwiegend körperlich

Vorwiegend geistig

Tägliche Arbeitszeit

Tägliche Überstunden (Res.)

$R^{2}$

\section{AV: Psychosomatische Beschwerden}

Alter (in Jahren)

$\begin{array}{lllll}0,02 & -0,02 & 0,10 & 0,09 & 0,02 \\ 0,06 & 0,01 & 0,13 * & 0,12 * & 0,06 \\ 0,12 * * & 0,06 & 0,19 * * & 0,09 & 0,13 *\end{array}$

Kind unter 18 im Haushalt

Anforderungsniveau - Referenz:

Helfer- und Anlerntätigkeit

Fachlich ausgerichtete Tätigkeiten

Komplexe Spezialistentätigkeiten

Hoch komplexe Tätigkeiten

Art der Tätigkeit - Referenz:

gleichermaßen geistig und körperlich

Vorwiegend körperlich

0,01

$-0,03$

$-0,01$

$-0,03$

$-0,02$

$-0,08$ **

$-0,14 * *$

0,06

$-0,06^{* *}$

$0,23 * *$

$0,25 * *$

$0,18^{* *}$

$0,25^{* *}$

$0,18 * *$

$0,20 * *$

0,10

$0,16^{* * *}$

$0,11 * *$

$0,12 * *$

$0,15 * *$

$0,23 * *$

$0,12 * *$

$-0,02$

$-0,01$

$0,10 * *$

$0,06 * *$

$-0,03$

$-0,03 *$

$-0,05 *$

0,05

$-0,03 *$

$-0,12 * *$

$-0,02$

$-0,01$

$-0,03$

$-0,16^{* *}$

$-0,08$

$-0,10$

$-0,14 * *$

$-0,05$

$-0,14 * *$

$-0,05$

$-0,13^{*}$

$-0,22 * *$

$-0,12$

$-0,22 * *$

$-0,05$

$-0,20 * *$

Vorwiegend geistig

Tägliche Arbeitszeit (direkt)

Tägliche Arbeitszeit (indirekt)

Tägliche Überstunden (Res.; direkt)

Tägliche Überstunden (Res.; indirekt)

Verkürzte Ruhezeiten

$R^{2}$

\section{AV: Work-Life-Balance}

Alter (in Jahren)

Kind unter 18 im Haushalt

Anforderungsniveau - Referenz:

Helfer- und Anlerntätigkeit

Fachlich ausgerichtete Tätigkeiten

Komplexe Spezialistentätigkeiten

Hoch komplexe Tätigkeiten

0,02

0,01

0,01

0,01

$-0,01$

$-0,03$

$-0,02$

$-0,02$

0,01

$0,03 *$

0,03

$0,09 * *$

0,09

$0,03 *$

$0,02 * *$

$0,02 * *$

$0,02 * *$

0,02

$0,01 * *$

0,10 **

0,11 **

0,16 **

0,10 ***

$0,01 * *$

$0,01 * *$

0,01 **

$0,05 * *$

$0,01 * *$

$0,07 * *$

$0,06^{* *}$

$0,09 * *$

$0,20 * *$

$0,05 * *$

$0,03 *$

$0,02 * *$

$0,04 * *$

$0,08 * *$

0,02 **

0,00

$-0,00$

0,02

$-0,02$

0,00

0,01

0,00

0,00

0,08

0,00

Art der Tätigkeit - Referenz:

gleichermaßen geistig und körperlich

Vorwiegend körperlich

Vorwiegend geistig

Tägliche Arbeitszeit (direkt)

Tägliche Arbeitszeit (indirekt)

Tägliche Überstunden (Res.; direkt)

0,06

0,06

$-0,02$

$0,13 *$

0,05

$-0,02$

0,06

$-0,00$

$0,13 *$

$-0,02$

0,00

0,09

$-0,01$

$-0,02$

Tägliche Überstunden (Res.; indirekt)

$\begin{array}{lllll}-0,04 * * & -0,04 * & -0,05 * & -0,04 & -0,04 * * \\ 0,11 * * & 0,13 * * & 0,09 * * & 0,01 & 0,09 * * \\ -0,14 * * & -0,14 * * & -0,13 * * & -0,05 & -0,15 * * \\ -0,02 * * & -0,02 * & -0,02 * * & -0,01 & -0,02 * * \\ -0,10 * * & -0,09 * * & -0,10 * * & -0,05 & -0,09 * * \\ -0,02 * * & -0,02 * * & -0,02 * * & -0,02 & -0,01 * *\end{array}$


Tab. A.5 (Fortsetzung)

Table A.5 (Continued)

\begin{tabular}{|c|c|c|c|c|c|}
\hline & Gesamt $(\beta)$ & Männer $(\beta)$ & Frauen $(\beta)$ & Schichtarbeit $(\beta)$ & $\begin{array}{l}\text { Keine Schichtarbeit } \\
(\beta)\end{array}$ \\
\hline Verkürzte Ruhezeiten & $-0,10 * *$ & $-0,09 * *$ & $-0,12 * *$ & $-0,07$ & $-0,08 * *$ \\
\hline$R^{2}$ & $0,06^{* *}$ & $0,06^{* *}$ & $0,08^{* *}$ & 0,02 & $0,06 * *$ \\
\hline $\begin{array}{l}\text { Korrelation Work-Life-Balan- } \\
\text { ce } \times \text { Psychosomatische Beschwerden }\end{array}$ & $-0,24 * *$ & $-0,21 * *$ & $-0,29 * *$ & $-0,22 * *$ & $-0,24 * *$ \\
\hline
\end{tabular}

Res Residuen

$* p<0,05 ; * * p<0,01$

Tab. A.6 Ergebnisse des Interaktionsmodells

Table A.6 Results of the interaction model

Gesamt $(\beta) \quad$ Männer $(\beta) \quad$ Frauen $(\beta) \quad$ Schichtarbeit $(\beta) \quad$ Keine Schichtarbeit
$(\beta)$

AV: Verkürzte Ruhezeiten

Alter (in Jahren)

Kind unter 18 im Haushalt

Anforderungsniveau - Referenz:

Helfer- und Anlerntätigkeit

Fachlich ausgerichtete Tätigkeiten

Komplexe Spezialistentätigkeiten

Hoch komplexe Tätigkeiten

Art der Tätigkeit - Referenz: gleichermaßen geistig und körperlich

Vorwiegend körperlich

Vorwiegend geistig

$R^{2}$

\section{AV: Psychosomatische Beschwerden}

Alter (in Jahren)

Kind unter 18 im Haushalt

Anforderungsniveau - Referenz:

Helfer- und Anlerntätigkeit

Fachlich ausgerichtete Tätigkeiten

Komplexe Spezialistentätigkeiten

Hoch komplexe Tätigkeiten

Art der Tätigkeit - Referenz:

gleichermaßen geistig und körperlich

Vorwiegend körperlich

Vorwiegend geistig

Tägliche Arbeitszeit

Tägliche Überstunden (Res.)

Verkürzte Ruhezeiten

Tägliche Arbeitszeit× verkürzte Ruhezeiten

$\begin{array}{lllll}-0,03 & -0,02 & -0,03 & -0,01 & -0,02 \\ 0,03 & 0,03 & 0,01 & -0,01 & 0,03 *\end{array}$

$-0,02$

$03^{*}$

$\begin{array}{lllll}0,05 & 0,01 & 0,13 * & 0,09 & 0,07\end{array}$

$0,10 * *$

$0,17 * *$

0,12

0,07

$0,23 * *$

$0,16^{* *}$

$0,29 * *$

$0,12 *$

$0,12 *$

$0,25 * *$

$\begin{array}{lllll}-0,03 * & -0,04 * & -0,00 & -0,03 & -0,03 * \\ -0,10^{* *} & -0,06^{* *} & -0,15^{* *} & 0,06 & -0,09 * *\end{array}$

$0,03 * * 0,02 * * 0,04 * * \quad 0,02 \quad 0,03 * *$

$\begin{array}{lllll}-0,02 & -0,01 & -0,05^{*} & 0,05 & -0,03\end{array}$

$\begin{array}{llll}-0,03 * & -0,00 & -0,01 & -0,04\end{array}$

$\begin{array}{lllll}-0,11 * * & -0,03 * * & -0,16^{*} & -0,08 & -0,09 \\ -0,14 * * & -0,05 & -0,14 * & -0,05 & -0,12 * \\ -0,22 * * & -0,13 * & -0,23 * * & -0,04 & -0,20 * *\end{array}$

Tägliche Überstunden (Res.) × verkürzte Ruhezeiten

$R^{2}$

\section{AV: Work-Life-Balance}

Alter (in Jahren)

Kind unter 18 im Haushalt

0,0

$-0,01$

0,02

0,01

0,02

0,01

$-0,01$

$-0,04$

$-0,01$

$-0,00$

0,01

$0,11 * *$

0,04

$0,11 * *$

0,10

$0,04 *$

$0,08 * *$

$0,07 * *$

$0,12 * *$

$0,15^{*}$

$0,11 * *$

$-0,03$

$0,07 * *$

$0,11 * *$

$0,20 * *$

$0,06 * *$

$-0,01$

$-0,01$

$-0,04$

$-0,00$

$-0,01$

$-0,00$

$-0,03$

0,02

$-0,02$

$0,03 * *$

$0,02 * *$

$0,04 * *$

$0,07 * *$

$0,02 * *$

0,01

0,00

0,02

$-0,02$

0,00

0,01

0,00

0,01

0,09

$-0,00$

Anforderungsniveau - Referenz:

Helfer- und Anlerntätigkeit

Fachlich ausgerichtete Tätigkeiten

Komplexe Spezialistentätigkeiten

\begin{tabular}{lllll}
0,05 & $-0,01$ & 0,10 & 0,05 & $-0,04$ \\
0,06 & 0,00 & $0,11^{*}$ & $-0,03$ & $-0,01$ \\
0,05 & 0,01 & 0,06 & 0,01 & $-0,04$ \\
\hline
\end{tabular}

Hoch komplexe Tätigkeiten 
Tab. A.6 (Fortsetzung)

Table A.6 (Continued)

Gesamt $(\beta) \quad$ Männer $(\beta) \quad$ Frauen $(\beta) \quad$ Schichtarbeit $(\beta) \quad$ Keine Schichtarbeit

$(\beta)$

Art der Tätigkeit - Referenz:

gleichermaßen geistig und körperlich

Vorwiegend körperlich

Vorwiegend geistig

$\begin{array}{ll}-0,05 * * & -0,04 * \\ 0,11 * * & 0,13 * * \\ -0,15^{* *} & -0,16^{* *} \\ -0,11 * * & -0,10^{* *} \\ -0,10^{* *} & -0,10^{* *} \\ 0,02 & 0,03 \\ 0,01 & 0,00\end{array}$

$-0,05$

$-0,05^{* *}$

Tägliche Arbeitszeit

Tägliche Überstunden (Res.)

Verkürzte Ruhezeiten

Tägl. Arbeitszeit× verkürzte Ruhezeiten

Tägliche Überstunden (Res.) × verkürzte Ruhezeiten

$R^{2}$

$\begin{array}{lllll}0,06 * * & 0,06 * * & 0,07 * * & 0,03 & 0,06 * * \\ -0,24 * * & -0,21 * * & -0,29 * * & -0,24 * * & -0,24 * *\end{array}$

Korrelation Work-Life-Balan-

ce $\times$ Psychosomatische Beschwerden

Res Residuen

$* p<0,05 ; * * p<0,01$

Tab. A.7 Ergebnisse der Modellvergleiche

Table A.7 Results of the model comparisons

\begin{tabular}{|c|c|c|c|c|c|c|c|}
\hline Modelle & Stichprobe & Abhängige Variable & $R_{1}^{2}$ & $R_{2}^{2}$ & $N$ & $F$-Wert $(d f 1, d f 2)$ & $p$-Wert \\
\hline \multirow{10}{*}{$\begin{array}{l}\text { Haupteffekt- } \\
\text { modell vs. } \\
\text { Null- } \\
\text { modell }\end{array}$} & Gesamt & \multirow{5}{*}{$\begin{array}{l}\text { Psychosomatische } \\
\text { Beschwerden }\end{array}$} & 0,03 & 0,01 & 6136 & $F(3,6125)=33,54$ & $<0,01$ \\
\hline & Männer & & 0,02 & 0,01 & 3854 & $F(3,3843)=10,46$ & $<0,01$ \\
\hline & Frauen & & 0,04 & 0,01 & 2282 & $F(3,2271)=21,20$ & $<0,01$ \\
\hline & Schichtarbeit & & 0,07 & 0,01 & 510 & $F(3,499)=10,89$ & $<0,01$ \\
\hline & Keine Schichtarbeit & & 0,02 & 0,01 & 5587 & $F(3,5576)=24,66$ & $<0,01$ \\
\hline & Gesamt & \multirow[t]{5}{*}{ Work-Life-Balance } & 0,06 & 0,02 & 6136 & $F(3,6125)=77,86$ & $<0,01$ \\
\hline & Männer & & 0,05 & 0,02 & 3854 & $F(3,3843)=44,69$ & $<0,01$ \\
\hline & Frauen & & 0,07 & 0,03 & 2282 & $F(3,2271)=33,27$ & $<0,01$ \\
\hline & Schichtarbeit & & 0,02 & 0,01 & 510 & $F(3,499)=1,36$ & 0,25 \\
\hline & Keine Schichtarbeit & & 0,06 & 0,02 & 5587 & $F(3,5576)=80,73$ & $<0,01$ \\
\hline \multirow{5}{*}{$\begin{array}{l}\text { Mediations- } \\
\text { modell vs. } \\
\text { Null- } \\
\text { modell }\end{array}$} & Gesamt & \multirow[t]{5}{*}{ Verkürzte Ruhezeiten } & 0,11 & 0,03 & 6136 & $F(2,6126)=274,09$ & $<0,01$ \\
\hline & Männer & & 0,12 & 0,02 & 3854 & $F(2,3844)=215,73$ & $<0,01$ \\
\hline & Frauen & & 0,10 & 0,04 & 2282 & $F(2,2272)=67,78$ & $<0,01$ \\
\hline & Schichtarbeit & & 0,06 & 0,02 & 510 & $F(2,500)=10,68$ & $<0,01$ \\
\hline & Keine Schichtarbeit & & 0,12 & 0,03 & 5587 & $F(2,5577)=292,19$ & $<0,01$ \\
\hline \multirow{10}{*}{$\begin{array}{l}\text { Interaktions- } \\
\text { modell vs. } \\
\text { Hauptef- } \\
\text { fektmodell }\end{array}$} & Gesamt & \multirow{5}{*}{$\begin{array}{l}\text { Psychosomatische } \\
\text { Beschwerden }\end{array}$} & 0,03 & 0,03 & 6136 & $F(2,6123)=9,46$ & $<0,01$ \\
\hline & Männer & & 0,02 & 0,02 & 3854 & $F(2,3841)=3,93$ & 0,02 \\
\hline & Frauen & & 0,04 & 0,04 & 2282 & $F(2,2269)=3,54$ & 0,03 \\
\hline & Schichtarbeit & & 0,07 & 0,07 & 510 & $F(2,497)=0,53$ & 0,59 \\
\hline & Keine Schichtarbeit & & 0,02 & 0,02 & 5587 & $F(2,5574)=2,85$ & 0,06 \\
\hline & Gesamt & \multirow[t]{5}{*}{ Work-Life-Balance } & 0,06 & 0,06 & 6136 & $F(2,6123)=6,50$ & $<0,01$ \\
\hline & Männer & & 0,06 & 0,05 & 3854 & $F(2,3841)=6,11$ & $<0,01$ \\
\hline & Frauen & & 0,07 & 0,07 & 2282 & $F(2,2269)=2,44$ & 0,09 \\
\hline & Schichtarbeit & & 0,03 & 0,02 & 510 & $F(2,497)=1,02$ & 0,36 \\
\hline & Keine Schichtarbeit & & 0,06 & 0,06 & 5587 & $F(2,5574)=5,92$ & $<0,01$ \\
\hline
\end{tabular}

$d f$ Freiheitsgrade 
Tab. A.8 Ergebnisse des Geschlechtervergleichs

Table A.8 Results of the gender comparisons

\begin{tabular}{|c|c|c|c|c|c|}
\hline Modell & Abhängige Variable & Unabhängige Variable & $\begin{array}{l}\text { Männer } \\
\beta \text { (SE) }\end{array}$ & $\begin{array}{l}\text { Frauen } \\
\beta(\mathrm{SE})\end{array}$ & $Z$ \\
\hline \multirow{7}{*}{$\begin{array}{l}\text { Hauptef- } \\
\text { fektmo- } \\
\text { dell }\end{array}$} & \multirow{3}{*}{$\begin{array}{l}\text { Psychosomatische } \\
\text { Beschwerden }\end{array}$} & Tägliche Arbeitszeit & $0,03(0,02)$ & $0,09(0,02)$ & $1,96^{*}$ \\
\hline & & Tägliche Überstunden & $0,06(0,02)$ & $0,11(0,02)$ & $1,69 *$ \\
\hline & & Verkürzte Ruhezeiten & $0,07(0,02)$ & $0,09(0,02)$ & 0,94 \\
\hline & \multirow[t]{3}{*}{ Work-Life-Balance } & Tägliche Arbeitszeit & $-0,14(0,02)$ & $-0,14(0,02)$ & 0,15 \\
\hline & & Tägliche Überstunden & $-0,09(0,02)$ & $-0,10(0,02)$ & $-0,45$ \\
\hline & & Verkürzte Ruhezeiten & $-0,09(0,02)$ & $-0,12(0,02)$ & $-1,15$ \\
\hline & \multicolumn{2}{|c|}{ Korrelation: Work-Life-Balance und psychosomatische Beschwerden } & $-0,21(0,02)$ & $-0,29(0,02)$ & $-3,34 * *$ \\
\hline \multirow{13}{*}{$\begin{array}{l}\text { Media- } \\
\text { tions- } \\
\text { modell }\end{array}$} & \multirow[t]{2}{*}{ Verkürzte Ruhezeiten } & Tägliche Arbeitszeit & $0,25(0,02)$ & $0,18(0,02)$ & $-2,98 * *$ \\
\hline & & Tägliche Überstunden & $0,20(0,02)$ & $0,15(0,02)$ & $-1,94 *$ \\
\hline & \multirow{5}{*}{$\begin{array}{l}\text { Psychosomatische } \\
\text { Beschwerden }\end{array}$} & Tägliche Arbeitszeit (direkt) & $0,03(0,02)$ & $0,09(0,02)$ & $1,92 *$ \\
\hline & & Tägliche Arbeitszeit (indirekt) & $0,02(0,00)$ & $0,02(0,00)$ & 0,00 \\
\hline & & Tägliche Überstunden (direkt) & $0,06(0,02)$ & $0,11(0,02)$ & $1,65 *$ \\
\hline & & Tägliche Überstunden (indirekt) & $0,01(0,00)$ & $0,01(0,00)$ & 0,18 \\
\hline & & Verkürzte Ruhezeiten & $0,06(0,02)$ & $0,09(0,02)$ & 1,04 \\
\hline & \multirow[t]{5}{*}{ Work-Life-Balance } & Tägliche Arbeitszeit (direkt) & $-0,14(0,02)$ & $-0,13(0,02)$ & 0,15 \\
\hline & & Tägliche Arbeitszeit (indirekt) & $-0,02(0,00)$ & $-0,02(0,00)$ & 0,00 \\
\hline & & Tägliche Überstunden (direkt) & $-0,09(0,02)$ & $-0,10(0,02)$ & $-0,38$ \\
\hline & & Tägliche Überstunden (indirekt) & $-0,02(0,00)$ & $-0,02(0,00)$ & $-0,18$ \\
\hline & & Verkürzte Ruhezeiten & $-0,09(0,02)$ & $-0,12(0,02)$ & $-1,26$ \\
\hline & \multicolumn{2}{|c|}{ Korrelation: Work-Life-Balance und psychosomatische Beschwerden } & $-0,21(0,02)$ & $-0,29(0,02)$ & $-3,30 * *$ \\
\hline \multirow{11}{*}{$\begin{array}{l}\text { Inter- } \\
\text { aktions- } \\
\text { modell }\end{array}$} & \multirow{5}{*}{$\begin{array}{l}\text { Psychosomatische } \\
\text { Beschwerden }\end{array}$} & Tägliche Arbeitszeit & $0,04(0,02)$ & $0,11(0,03)$ & $1,93^{*}$ \\
\hline & & Tägliche Überstunden & $0,07(0,02)$ & $0,12(0,03)$ & 1,59 \\
\hline & & Verkürzte Ruhezeiten & $0,07(0,02)$ & $0,11(0,03)$ & 1,35 \\
\hline & & $\begin{array}{l}\text { Interaktion verkürzte Ruhezeiten } \times \text { tägliche } \\
\text { Arbeitszeit }\end{array}$ & $-0,01(0,03)$ & $-0,04(0,03)$ & $-0,82$ \\
\hline & & $\begin{array}{l}\text { Interaktion verkürzte Ruhezeiten } \times \text { tägliche } \\
\text { Überstunden }\end{array}$ & $-0,00(0,02)$ & $-0,03(0,03)$ & $-0,71$ \\
\hline & \multirow[t]{5}{*}{ Work-Life-Balance } & Tägliche Arbeitszeit & $-0,16(0,02)$ & $-0,12(0,03)$ & 0,98 \\
\hline & & Tägliche Überstunden & $-0,10(0,02)$ & $-0,11(0,03)$ & $-0,49$ \\
\hline & & Verkürzte Ruhezeiten & $-0,10(0,02)$ & $-0,12(0,02)$ & $-0,69$ \\
\hline & & $\begin{array}{l}\text { Interaktion verkürzte Ruhezeiten } \times \text { tägliche } \\
\text { Arbeitszeit }\end{array}$ & $0,03(0,03)$ & $-0,02(0,03)$ & $-1,20$ \\
\hline & & $\begin{array}{l}\text { Interaktion verkürzte Ruhezeiten } \times \text { tägliche } \\
\text { Überstunden }\end{array}$ & $0,00(0,02)$ & $0,03(0,03)$ & 0,76 \\
\hline & \multicolumn{2}{|c|}{ Korrelation: Work-Life-Balance und psychosomatische Beschwerden** } & $-0,21(0,02)$ & $-0,29(0,02)$ & $-2,93 * *$ \\
\hline
\end{tabular}

SE Standardfehler für $\beta$

$* p<0,05$; ** $p<0,01$ 
Tab. A.9 Ergebnisse des Vergleichs zwischen Beschäftigten mit und ohne Schichtarbeit

Table A.9 Results of the comparisons between employees working in shifts versus not working in shifts

\begin{tabular}{|c|c|c|c|c|c|}
\hline \multirow[t]{2}{*}{ Modell } & \multirow[t]{2}{*}{ Abhängige Variable } & \multirow[t]{2}{*}{ Unabhängige Variable } & \multirow{2}{*}{$\begin{array}{l}\text { Schichtarbeit } \\
\beta \text { (SE) }\end{array}$} & \multirow{2}{*}{$\begin{array}{l}\text { Keine Schichtar- } \\
\text { beit } \\
\beta(\mathrm{SE})\end{array}$} & \multirow[t]{2}{*}{$Z$} \\
\hline & & & & & \\
\hline \multirow{7}{*}{$\begin{array}{l}\text { Hauptef- } \\
\text { fektmo- } \\
\text { dell }\end{array}$} & \multirow{3}{*}{$\begin{array}{l}\text { Psychosomatische } \\
\text { Beschwerden }\end{array}$} & Tägliche Arbeitszeit & $0,09(0,05)$ & $0,03(0,01)$ & $-1,20$ \\
\hline & & Tägliche Überstunden & $0,16(0,05)$ & $0,10(0,01)$ & $-1,30$ \\
\hline & & Verkürzte Ruhezeiten & $0,20(0,04)$ & $0,05(0,01)$ & $-3,31 * *$ \\
\hline & \multirow[t]{3}{*}{ Work-Life-Balance } & Tägliche Arbeitszeit & $-0,06(0,05)$ & $-0,15(0,01)$ & $-1,92 *$ \\
\hline & & Tägliche Überstunden & $-0,05(0,05)$ & $-0,09(0,01)$ & $-0,79$ \\
\hline & & Verkürzte Ruhezeiten & $-0,07(0,05)$ & $-0,09(0,01)$ & $-0,29$ \\
\hline & \multicolumn{2}{|c|}{ Korrelation: Work-Life-Balance und psychosomatische Beschwerden } & $-0,22(0,04)$ & $-0,24(0,01)$ & $-0,41$ \\
\hline \multirow{13}{*}{$\begin{array}{l}\text { Media- } \\
\text { tions- } \\
\text { modell }\end{array}$} & \multirow[t]{2}{*}{ Verkürzte Ruhezeiten } & Tägliche Arbeitszeit & $0,10(0,05)$ & $0,25(0,01)$ & $3,08 * *$ \\
\hline & & Tägliche Überstunden & $0,23(0,05)$ & $0,16(0,01)$ & $-1,35$ \\
\hline & \multirow{5}{*}{$\begin{array}{l}\text { Psychosomatische } \\
\text { Beschwerden }\end{array}$} & Tägliche Arbeitszeit (direkt) & $0,09(0,05)$ & $0,03(0,01)$ & $-1,18$ \\
\hline & & Tägliche Arbeitszeit (indirekt) & $0,02(0,01)$ & $0,01(0,00)$ & $-0,60$ \\
\hline & & Tägliche Überstunden (direkt) & $0,16(0,05)$ & $0,10(0,01)$ & $-1,62$ \\
\hline & & Tägliche Überstunden (indirekt) & $0,05(0,01)$ & $0,01(0,00)$ & $-2,62 * *$ \\
\hline & & Verkürzte Ruhezeiten & $0,20(0,04)$ & $0,05(0,01)$ & $-0,76$ \\
\hline & \multirow[t]{5}{*}{ Work-Life-Balance } & Tägliche Arbeitszeit (direkt) & $-0,05(0,05)$ & $-0,15(0,01)$ & $-1,92 *$ \\
\hline & & Tägliche Arbeitszeit (indirekt) & $-0,01(0,01)$ & $-0,02(0,00)$ & $-1,94 *$ \\
\hline & & Tägliche Überstunden (direkt) & $-0,05(0,05)$ & $-0,09(0,01)$ & $-0,79$ \\
\hline & & Tägliche Überstunden (indirekt) & $-0,02(0,01)$ & $-0,01(0,00)$ & $-0,43$ \\
\hline & & Verkürzte Ruhezeiten & $-0,07(0,05)$ & $-0,08(0,01)$ & $-0,23$ \\
\hline & \multicolumn{2}{|c|}{ Korrelation: Work-Life-Balance und psychosomatische Beschwerden } & $-0,22(0,04)$ & $-0,24(0,01)$ & $-0,43$ \\
\hline \multirow{11}{*}{$\begin{array}{l}\text { Inter- } \\
\text { aktions- } \\
\text { modell }\end{array}$} & \multirow{5}{*}{$\begin{array}{l}\text { Psychosomatische } \\
\text { Beschwerden }\end{array}$} & Tägliche Arbeitszeit & $0,10(0,04)$ & $0,04(0,02)$ & $-1,31$ \\
\hline & & Tägliche Überstunden & $0,15(0,06)$ & $0,11(0,02)$ & $-0,68$ \\
\hline & & Verkürzte Ruhezeiten & $0,20(0,05)$ & $0,06(0,02)$ & $-2,90 * *$ \\
\hline & & $\begin{array}{l}\text { Interaktion verkürzte Ruhezeiten } \times \text { tägliche } \\
\text { Arbeitszeit }\end{array}$ & $0,00(0,05)$ & $-0,01(0,02)$ & $-0,21$ \\
\hline & & $\begin{array}{l}\text { Interaktion verkürzte Ruhezeiten } \times \text { tägliche } \\
\text { Überstunden }\end{array}$ & $0,02(0,05)$ & $-0,02(0,02)$ & $-0,71$ \\
\hline & \multirow[t]{5}{*}{ Work-Life-Balance } & Tägliche Arbeitszeit & $-0,01(0,06)$ & $-0,16(0,02)$ & $-2,39 * *$ \\
\hline & & Tägliche Überstunden & $0,00(0,06)$ & $-0,11(0,02)$ & $-1,69 *$ \\
\hline & & Verkürzte Ruhezeiten & $-0,06(0,05)$ & $-0,09(0,02)$ & $-0,73$ \\
\hline & & $\begin{array}{l}\text { Interaktion verkürzte Ruhezeiten } \times \text { tägliche } \\
\text { Arbeitszeit }\end{array}$ & $-0,06(0,06)$ & $0,02(0,02)$ & 1,22 \\
\hline & & $\begin{array}{l}\text { Interaktion verkürzte Ruhezeiten } \times \text { tägliche } \\
\text { Überstunden }\end{array}$ & $-0,06(0,06)$ & $0,02(0,02)$ & 1,29 \\
\hline & \multicolumn{2}{|c|}{ Korrelation: Work-Life-Balance und psychosomatische Beschwerden** } & $-0,24(0,04)$ & $-0,24(0,01)$ & $-0,02$ \\
\hline
\end{tabular}

$S E$ Standardfehler für $\beta$

$* p<0,05 ; * * p<0,01$

\section{Literatur}

Åkerstedt T, Kecklund G (2017) What work schedule characteristics constitute a problem to the individual? A representative study of Swedish shift workers. Appl Ergon 59:320-325

Amlinger-Chatterjee M (2016) Psychische Gesundheit in der Arbeitswelt: Atypische Arbeitszeiten (baua: Bericht). Bundesanstalt für Arbeitsschutz und Arbeitsmedizin, Dortmund, Berlin, Dresden (20.10.2018 unter https://www.baua.de/DE/Angebote/ Publikationen/Berichte/F2353-3a.html)

Amlinger-Chatterjee M, Wöhrmann AM (2017) Flexible Arbeitszeiten. Z Arb Wiss 71:39-51
Arlinghaus A (2017) Wissensarbeit: Aktuelle arbeitswissenschaftliche Erkenntnisse. MBF-Report Nr. 35. Hans-Böckler-Stiftung, Düsseldorf (Zugriff am 18.04.2018 unter https://www.boeckler.de/ pdf/p_mbf_report_2017_35.pdf)

Arlinghaus A, Lott Y (2018) Schichtarbeit gesund und sozialverträglich gestalten. Forschungsförderung Report Nr. 3. Hans-BöcklerStiftung, Düsseldorf (Zugriff am 08.03.2018 unter https://www. boeckler.de/pdf/p_fofoe_report_003_2018.pdf)

Arlinghaus A, Nachreiner F (2012) Arbeit zu unüblichen Zeiten Arbeit mit unüblichem Risiko. Z Arb Wiss 66:291-305

Backhaus N, Brenscheidt F, Tisch A (2019) Verkürzte Ruhezeiten bei Vollzeitbeschäftigten: Ergebnisse aus der BAuA-Arbeitszeitbefragung 2017. In: GfA (Hrsg) Frühjahrskongress 2019: Arbeit 
interdisziplinär analysieren - bewerten - gestalten. Gesellschaft für Arbeitswissenschaft, Dortmund (Beitrag C.1.14.)

Backhaus N, Tisch A, Wöhrmann AM (2018) BAuA-Arbeitszeitbefragung: Vergleich 2015-2017 (baua: Bericht). Bundesanstalt für Arbeitsschutz und Arbeitsmedizin, Dortmund/Berlin/Dresden ( $\mathrm{Zu}-$ griff am 27.09.2018 unter www.baua.de/dok/8752798)

BAuA (2016) Arbeitszeitreport Deutschland 2016 (baua: Bericht). Bundesanstalt für Arbeitsschutz und Arbeitsmedizin, Dortmund, Berlin, Dresden (Zugriff am 03.03.2017 unter www.baua.de/dok/ 8137556)

BDA (2015) Arbeitswelt 4.0 - Chancen nutzen, Herausforderungen meistern: Positionen der BDA zum Grünbuch „Arbeiten 4.0“ des Bundesministeriums für Arbeit und Soziales. Bundesvereinigung der Deutschen Arbeitgeberverbände, Berlin

Beermann B, Amlinger-Chatterjee M, Brenscheidt F, Gerstenberg S, Niehaus M, Wöhrmann AM (2017) Orts- und zeitflexibles Arbeiten: Gesundheitliche Chancen und Risiken (baua: Bericht). Bundesanstalt für Arbeitsschutz und Arbeitsmedizin, Dortmund, Berlin, Dresden (Zugriff am 19.04.2018 unter www.baua.de/dok/ 8729000)

Bernstrøm VH, Alves DE, Ellingsen D, Ingelsrud MH (2019) Healthy working time arrangements for healthcare personnel and patients: a systematic literature review. BMC Health Serv Res 19:193

Böhne A, Breutmann N (2012) Flexibilisierung der Arbeitswelt aus Unternehmenssicht: Chancen und Risiken für Arbeitgeber und Arbeitnehmer. In: Badura B, Ducki A, Schröder H, Klose J, Meyer M (Hrsg) Fehlzeiten-Report 2012: Gesundheit in der flexiblen Arbeitswelt: Chancen nutzen - Risiken minimieren. Springer, Berlin, Heidelberg, S 23-27

Börkircher M, Walleter R (2018) Digitalisierung, Industrie und Arbeit 4.0 aus Sicht der Verbände der Metall- und Elektroindustrie. In: Cernavin O, Schröter W, Stowasser S (Hrsg) Prävention 4.0 : Analysen und Handlungsempfehlungen für eine produktive und gesunde Arbeit 4.0. Springer, Wiesbaden, S 67-79

Bundesagentur für Arbeit (2015) Klassifikation der Berufe 2010. Bundesagentur für Arbeit, Nürnberg (Zugriff am 14.05.2018 unter https://statistik.arbeitsagentur.de/Navigation/Statistik/Grundlagen/ Klassifikation-der-Berufe/KldB2010/KldB2010-Nav.html)

Clogg CC, Petkova E, Haritou A (1995) Statistical methods for comparing regression coefficients between models. Am J Sociol 100:1261-1293

Costa G (2010) Shift work and health: current problems and preventive actions. Saf Health Work 1:112-123

Dahlgren A, Tucker P, Gustavsson P, Rudman A (2016) Quick returns and night work as predictors of sleep quality, fatigue, work-family balance and satisfaction with work hours. Chronobiol Int 33:759-767

Deutscher Bundestag (2018) Entwurf eines Gesetzes zur Änderung des Arbeitszeitgesetzes (Drucksache Nr. 19/1174). Deutscher Bundestag, Berlin (Zugriff am 22.11.2018 unter http://dip21. bundestag.de/dip21/btd/19/011/1901174.pdf)

Eldevik MF, Flo E, Moen BE, Pallesen S, Bjorvatn B (2013) Insomnia, excessive sleepiness, excessive fatigue, anxiety, depression and shift work disorder in nurses having less than 11 hours in-between shifts. Plos One 8:e70882

Fagan C, Lyonette C, Smith M, Saldaña-Tejeda A (2012) The influence of working time arrangements on work-life integration or 'balance': a review of the international evidence. International Labour Office, Geneva

Flo E, Pallesen S, Moen BE, Waage S, Bjorvatn B (2014) Short rest periods between work shifts predict sleep and health problems in nurses at 1-year follow-up. Occup Environ Med 71:555-561

Folkard S, Lombardi DA (2006) Modeling the impact of the components of long work hours on injuries and "accidents". Am J Ind Med 49:953-963

Franke F (2015) Is Work Intensification Extra Stress? J Pers Psychol $14: 17-27$
Gilbert-Ouimet M, Ma H, Glazier R, Brisson C, Mustard C, Smith PM (2018) Adverse effect of long work hours on incident diabetes in 7065 Ontario workers followed for 12 years. BMJ Open Diabetes Res Care 6:e496

Greene WH (2003) Econometric analysis, 5. Aufl. Pearson Education, Upper Saddle River, NJ

Greubel J, Arlinghaus A, Nachreiner F, Lombardi DA (2016) Higher risks when working unusual times? A cross-validation of the effects on safety, health, and work-life balance. Int Arch Occup Environ Health 89:1205-1214

Häring A, Schütz H, Middendorf L, Hausen J, Brauner C, Wöhrmann AM (2018) Methodenbericht und Fragebogen zur BAuAArbeitszeitbefragung 2017 (baua: Bericht). Bundesanstalt für Arbeitsschutz und Arbeitsmedizin, Dortmund, Berlin, Dresden (Zugriff am 23.09.2018 unter https://www.baua.de/DE/Angebote/ Publikationen/Berichte/F2398-3.html)

Härmä M, Ropponen A, Hakola T, Koskinen A, Vanttola P, Puttonen S, Kivimäki $M$ et al (2015) Developing register-based measures for assessment of working time patterns for epidemiologic studies. Scand J Work Environ Health 41:268-279

Härmä M, Karhula K, Ropponen A, Puttonen S, Koskinen A, Ojajärvi A, Kivimäki M et al (2018) Association of changes in work shifts and shift intensity with change in fatigue and disturbed sleep: a within-subject study. Scand J Work Environ Health 44:394-402. https://doi.org/10.5271/sjweh.3730

Hassler M, Rau R, Hupfeld J, Paridon H (2016) Auswirkungen von ständiger Erreichbarkeit und Präventionsmöglichkeiten. Teil 2: Eine wissenschaftliche Untersuchung zu potenziellen Folgen für Erholung und Gesundheit und Gestaltungsvorschläge für Unternehmen. iga.report Nr. 23. Initiative Gesundheit und Arbeit, Berlin (Zugriff am 23.04.2018 unter https://www.iga-info.de/ veroeffentlichungen/igareporte/igareport-23-teil-2/)

Itani O, Kaneita Y (2016) The association between shift work and health: a review. Sleep Biol Rhythms 14:231-239

Karhula K, Koskinen A, Ojajärvi A, Ropponen A, Puttonen S, Kivimäki M, Härmä M (2018) Are changes in objective working hour characteristics associated with changes in work-life conflict among hospital employees working shifts? A 7-year follow-up. Occup Environ Med 75:407-411

Karhula K, Puttonen S, Ropponen A, Koskinen A, Ojajärvi A, Kivimäki M, Härmä M (2017) Objective working hour characteristics and work-life conflict among hospital employees in the Finnish public sector study. Chronobiol Int 34:876-885

Kecklund G, Åkerstedt T (1995) Effects of timing of shifts on sleepiness and sleep duration. J Sleep Res 4:47-50

Kim B-M, Lee B-E, Park H-S, Kim Y-J, Suh Y-J, Kim J-Y, Ha E-H et al (2016) Long working hours and overweight and obesity in working adults. Ann of Occup and Environ Med 28:36

Kivimäki M, Jokela M, Nyberg ST, Singh-Manoux A, Fransson EI, Alfredsson L, Virtanen M et al (2015) Long working hours and risk of coronary heart disease and stroke: a systematic review and meta-analysis of published and unpublished data for 603838 individuals. Lancet 386:1739-1746

Kivimäki M, Nyberg ST, Batty GD, Kawachi I, Jokela M, Alfredsson L, Tabak AG et al (2017) Long working hours as a risk factor for atrial fibrillation: a multi-cohort study. Eur Heart $\mathrm{J}$ 38:2621-2628

Landtag Nordrhein-Westfalen (2018) Gute Arbeitszeiten sichern Schutzrechte der Beschäftigten stärken - Die Digitalisierung der Arbeitswelt gestalten! (Drucksache Nr. 17/1665). Landtag Nordrhein-Westfalen, Düsseldorf (Zugriff am 22.11.2018 unter https://www.landtag.nrw.de/portal/WWW/dokumentenarchiv/ Dokument/MMD17-1665.pdf)

Li CY, Sung FC (1999) A review of the healthy worker effect in occupational epidemiology. Occup Med 49:225-229

Lott Y (2018) Does flexibility help employees switch off from work? Flexible working-time arrangements and cognitive work-to-home 
spillover for women and men in Germany. Soc Indic Res. https:// doi.org/10.1007/s11205-018-2031-z

Meijman TF, Mulder G (1998) Psychological aspects of workload. In: Drenth PJD, Thierry H, de Wolff CJ (Hrsg) Handbook of work and organizational psychology. Psychology Press, Hove, S 5-34

Miilunpalo S, Vuori I, Oja P, Pasanen M, Urponen H (1997) Self-rated health status as a health measure: the predictive value of self-reported health status on the use of physician services and on mortality in the working-age population. J Clin Epidemiol 50:517-528

Müller G, Tisch A, Wöhrmann AM (2018) The impact of long working hours on the health of German employees. Ger J Hum Resour Manage 32:217-235

Muthén LK, Muthén BO (2015) Mplus user's guide, 7. Aufl. Muthén \& Muthén, Los Angeles

Ng TWH, Feldman DC (2008) Long work hours: a social identity perspective on meta-analysis data. J Organ Behav 29:853-880

Nielsen HB, Hansen ÅM, Conway SH, Dyreborg J, Hansen J, Kolstad HA, Garde AH et al (2019) Short time between shifts and risk of injury among Danish hospital workers: a register-based cohort study. Scand J Work Environ Health 45:166-173

Pangert B, Pauls N, Schüpbach H (2016) Die Auswirkungen arbeitsbezogener erweiterter Erreichbarkeit auf Life-Domain-Balance und Gesundheit (baua: Bericht). Bundesanstalt für Arbeitsschutz und Arbeitsmedizin, Dortmund/Berlin/Dresden (Zugriff am 19.04.2018 unter https://www.baua.de/DE/Angebote/ Publikationen/Berichte/Gd76.html)

Pauls N, Schlett C, Pangert B, Menz W (2019) Den Umgang mit arbeitsbezogener erweiterter Erreichbarkeit gesund gestalten. In: Rump J, Eilers S (Hrsg) Arbeitszeitpolitik: Zielkonflikte in der betrieblichen Arbeitszeitgestaltung lösen. Springer, Berlin, Heidelberg, S 197-211

Presser HB (2005) Embracing complexity: work schedules and family life in a 24/7 economy. In: Bianchi SM, Casper LM, Berkowitz KR (Hrsg) Work, family, health, and well-being. Lawrence Erlbaum, Mahwah, S 43-48

Puttonen S, Härmä M, Hublin C (2010) Shift work and cardiovascular disease: pathways from circadian stress to morbidity. Scand J Work Environ Health 36:96-108

Rothe I, Beermann B, Wöhrmann AM (2017) Arbeitswissenschaftliche Erkenntnisse zu Arbeitszeit und Gesundheit. In: Schröder L, Urban H-J (Hrsg) Gute Arbeit: Streit um Zeit - Arbeitszeit und Gesundheit. Bund-Verlag, Frankfurt am Main, S 123-135

Schlick C, Bruder R, Luczak H (2018) Arbeitswissenschaft, 4. Aufl. Springer Vieweg, Berlin, Heidelberg

Sonnentag S, Fritz C (2007) The recovery experience questionnaire: development and validation of a measure for assessing recuperation and unwinding from work. J Occup Health Psychol $12: 204-221$
Sonnentag S, Natter E (2004) Flight attendants' daily recovery from work: Is there no place like home? Int J Stress Manag 11:366-391

Vedaa $\varnothing$, Mørland E, Larsen M, Harris A, Erevik E, Sivertsen B, Pallesen $\mathrm{S}$ et al (2017a) Sleep detriments associated with quick returns in rotating shift work: a diary study. J Occup Environ Med 59:522-527

Vedaa Ø, Pallesen S, Waage S, Bjorvatn B, Sivertsen B, Erevik E, Harris A et al (2017b) Short rest between shift intervals increases the risk of sick leave: a prospective registry study. Occup Environ Med 74:496-501

Vedaa $\varnothing$, Harris A, Erevik EK, Waage S, Bjorvatn B, Sivertsen B, Pallesen $S$ et al (2019) Short rest between shifts (quick returns) and night work is associated with work-related accidents. Int Arch Occup Environ Health. https://doi.org/10.1007/s00420-01901421-8

Vedaa $\emptyset$, Harris A, Bjorvatn B, Waage S, Sivertsen B, Tucker P, Pallesen S (2016) Systematic review of the relationship between quick returns in rotating shift work and health-related outcomes. Ergonomics 59:1-14

Virtanen M, Jokela M, Nyberg ST, Madsen IEH, Lallukka T, Ahola K, Kivimäki M et al (2015) Long working hours and alcohol use: systematic review and meta-analysis of published studies and unpublished individual participant data. BMJ 350:g7772

Virtanen M, Jokela M, Madsen IE, Magnusson Hanson LL, Lallukka T, Nyberg ST, Kivimäki M et al (2018) Long working hours and depressive symptoms: systematic review and meta-analysis of published studies and unpublished individual participant data. Scand J Work Environ Health 44(3):239-250

Vogel M, Braungardt T, Meyer W, Schneider W (2012) The effects of shift work on physical and mental health. J Neural Transm 119:1121-1132

Wendsche J, Lohmann-Haislah A (2016) Psychische Gesundheit in der Arbeitswelt - Pausen (baua: Bericht). Bundesanstalt für Arbeitsschutz und Arbeitsmedizin, Dortmund, Berlin, Dresden (Zugriff am 20.03.2018 unter https://www.baua.de/DE/Angebote/ Publikationen/Berichte/F2353-3b.pdf)

Wirtz A (2010) Gesundheitliche und soziale Auswirkungen langer Arbeitszeiten. Bundesanstalt für Arbeitsschutz und Arbeitsmedizin, Dortmund, Berlin, Dresden

Wu S, Wang R, Zhao Y, Ma X, Wu M, Yan X, He J (2013) The relationship between self-rated health and objective health status: a population-based study. BMC Public Health 13:320

Zijlstra FRH, Sonnentag S (2006) After work is done: psychological perspectives on recovery from work. Eur J Work Organ Psychol 15:129-138 